Posenato, D., Kripakaran, P., Inaudi, D. and Smith, I.F.C. "Methodologies for model-free data interpretation of civil engineering structures" Computers and Structures, Vol 88, No 7/8, 2010, pp 467-482. doi:10.1016/j.compstruc.2010.01.001

\title{
Methodologies for model-free data interpretation of civil engineering structures
}

Daniele Posenato ${ }^{1}$, Prakash Kripakaran ${ }^{2}$, Daniele Inaudi ${ }^{1}$ and Ian F.C. Smith ${ }^{3}$

${ }^{1}$ Smartec SA, via Pobiette 11, CH-6928 Manno, Switzerland

${ }^{2}$ Ecole Polytechnique Fédérale de Lausanne (EPFL), Station 18,

CH-1015 Lausanne, Switzerland

${ }^{3}$ Corresponding author: Ecole Polytechnique Fédérale de Lausanne (EPFL), Station 18,

CH-1015 Lausanne, Switzerland. Ph: (+41) 21693 5242, Fax: (+41) 21693 4748, Ian.Smith@epfl.ch

\author{
Abstract \\ Structural Health Monitoring (SHM) has the potential to provide quantitative and reliable \\ data on the real condition of structures, observe the evolution of their behaviour and detect \\ degradation. This paper presents two methodologies for model-free data interpretation to \\ identify and localize anomalous behaviour in civil engineering structures. Two statistical \\ methods based on i) moving principal component analysis and ii) robust regression analysis \\ are demonstrated to be useful for damage detection during continuous static monitoring of \\ civil structures. The methodologies are tested on numerically simulated elements with \\ sensors for a range of noise in measurements. A comparative study with other statistical \\ analyses demonstrates superior performance of these methods for damage detection. \\ Approaches for accommodating outliers and missing data, which are commonly \\ encountered in structural health monitoring for civil structures, are also proposed. To \\ ensure that the methodologies are scalable for complex structures with many sensors, a \\ clustering algorithm groups sensors that have strong correlations between their
}


measurements. Methodologies are then validated on two full-scale structures. The results show the ability of the methodology to identify abrupt permanent changes in behavior.

Keywords: Structural health monitoring, data interpretation, signal processing, pattern recognition, principal component analyses, clustering

\section{Introduction}

The collapse of a structure deters the public confidence in safety of the infrastructure they use every day. Once in use, each structure is subjected to evolving patterns of loads and other actions. Often the intensity and type of these actions are very different from those taken into account during design. The sum of these uncertainties created during design, construction and use poses a great challenge to the engineers and institutions in charge of structural safety, maintenance and operation [1]. For these reasons, the number of structures that are being monitored is rapidly growing. The most difficult challenge that faces the Structural Health Monitoring (SHM) community is not the lack of measurement technology but rather, finding rational methods to acquire, process, and analyze large amounts of data in order to extract useful information related to the health of structures [2-3]. Despite continuous evolution of research, for continuous static monitoring, no reliable strategies for identifying damage have been proposed and verified for broad classes of civil structures.

Moyo and Brownjohn [4] proposed a detection of anomalous structural behaviour using discrete wavelet analysis. Experimental studies on structural damage identification based 
on wavelet packet transform have been done with good results [5-7]. Other techniques widely used are based on autoregressive methodologies. They first estimate the relations between sensors and all their previous measurements, and then identify new events based on the evolution of the parameters of the autoregressive model [8-13]. Deraemaeker et al. [14] presented a methodology for damage detection under changing environmental conditions. The effects of environment are treated using factor analysis and damage is detected using statistical process control with the multivariate Shewhart-T control charts. Posenato et al. [15] proposed a methodology based on Moving Principal Component Analysis (MPCA). After comparing several algorithms for different levels of damage, Posenato et al. [15] selected MPCA as the best algorithm.

The majority of the data-interpretation methods studied for continuous monitoring fail to include practical complications in the data set such as variations due to daily traffic and measurement noise. Simple external events such as traffic, environmental changes or other short term actions may mask the presence of damage or also result in false alarms. Sensors also involve noise in measurements. Large levels of noise can render data-interpretation algorithms ineffective [16-18].

Many algorithms also require that the measurement series is stationary and complete. However, collected time series are often incomplete due to the nature of long-term static monitoring [19]. For example, periodic maintenance of the system can generate interruptions of measurements and consequently create big gaps in the time series. Missing values can also be due to instrument failure, extreme natural phenomena, power 
interruptions or simply due to removal of erroneous measurements. Incomplete data sets significantly distort results from data interpretation. Therefore, data preparation is extremely critical to take care of missing values. Data imputation methods have been shown to perform better than list-wise deletion or pair-wise deletion [20-21]. Several techniques have been proposed to generate replacement values for the missing data. For example, Wei and Tang [19] proposed a generic framework for missing data using neural networks. A two-stage filling algorithm was implemented. One drawback of this algorithm is that it requires a long training period due to the use of a neural network. Other techniques based on k-Nearest Neighbour method [22-25] and regression [26-27], can be used only in case of Missing at Random (MAR) and not in case of Missing Completely at Random (MCAR). Techniques based on autoregressive analysis [28-29] can detect trends in data. However, they are not useful for damage detection.

Outliers may strongly and misleadingly affect conclusions of widely used methodologies for damage detection. Outliers can be either removed and the datasets can be handled using methods for managing missing data, or they can be replaced by appropriate values [30-31]. Penny and Jolliffe [32] conduct a comparison study on several multivariate outlier detection methods and the results indicate that no technique is superior to all others. A detailed review on the most recent algorithms for managing missing data have been done by BenGal [33] and Yenduri and Iyengar [34].

The decreasing cost of sensors is causing an increase in the number of monitored structures. Most monitored structures have sensors that measure several types of parameters. Examples 
are applications such as the Götaälvbron bridge in Sweden where more than 70000 sensors are installed in a single bridge (55000 strain sensors and 11000 temperature sensors) [35] and the new I35 bridge in Minneapolis with more than 350 sensors (150 strain gauge vibrating wires, 150 thermocouples, 10 potentiometers, 20 accelerometers, 4 corrosions, 12 long gauge optical fibres,...) [1][36]. Analyzing databases with large amounts of heterogeneous data is a complex task. Implementing a methodology to process and analyze all data could be critical in terms of computation time. The majority of algorithms, in particular those based on autoregressive analysis, are applicable only on time series from a few sensors; Clustering techniques may help through dividing the problem into classes of inferior complexity [37]. To the authors’ knowledge, a methodology that handles these complexities without manual intervention is currently unavailable.

This paper presents a methodology for automatically detecting structural damage. Damage is assumed to result in abrupt and permanent changes in datasets obtained from long-term static monitoring. This methodology is also shown to be suitable for handling noise, outliers and incomplete data. The research builds upon previous work by Posenato et al. [15] on MPCA for measurement data interpretation. In addition, when there are many sensors, they can be organized into clusters to reduce dimensionality.

The paper is organized as follows: Section 2 provides a short description of the model-free data analysis. Section 3 describes the numerical simulation used to compare the damage detection performances of several algorithms and describes the damage detection methodologies. Section 4 presents a comparative study between MPCA and other methods. 
Section 5 presents how MPCA can be generalized and applied in cases of missing data and outliers. This section introduces a clustering-with-overlapping algorithm based on the correlation between the sensors. Section 6 presents examples of the applications of the proposed methodology to full-scale structures.

\section{Model-free data-interpretation methods}

Static monitoring can lead to damage identification by comparing static structural response (displacements or strains due to environmental effects and applied loading) with predictions from behaviour models. For each structure, a team of experts is required to analyze the data and develop a precise model to validate the results. Structural models are difficult to create and may not accurately reflect real behaviour. Difficulties and uncertainties increase in presence of complex civil structures so that well-defined and unique behaviour models of structures cannot be clearly identified. Furthermore, model based structural identification may not succeed in identifying the right damage.

Another approach is to evaluate data statistically. This approach involves examining changes in time series over time. The methodology is completely data-driven, i.e., the analysis of data is done without any information of the structure. Therefore the methodology is applicable to many types of structures. Practitioners have difficulties in choosing the best algorithm from the numerous algorithms available for signal processing. Posenato et al. [15] compared several algorithms for different levels of damage scenarios. The comparative study showed that for quasi-static monitoring of civil structures, new 
methodologies such as MPCA perform better than wavelet methods, Short Term Fourier Transform and Instance-Based Method. Few other comparisons have been done for these methods.

Typical practical applications are, however, more complex than studied by Posenato et al. [15], Nair et al. [12], Brownjohn et al. [38] and Omenzetter et al. [39]. More specifically the varying sensor noise levels, presence of outliers or missing data and finally the number, types and location of sensors may preclude the simultaneous interpretation. Evaluating the performance of the algorithms with respect to these aspects can lead to the selection of the robust methods for interpretation of measurements from continuous monitoring.

The following algorithms are compared in this paper.

- Auto Regressive with Moving Average (ARMA) [2][9][12][40] ;

- Box-Jenkins (BJ) [4];

- Seasonal ARIMA [10];

- Discrete Wavelet Transform (DWT) [4][41];

- Wavelet Packet Transform (WPT) [5][6-7][42];

- Robust Regression Analysis (RRA) [43-44];

- Instance Based Method (IBM) [15][46-47] ;

- Correlation Anomaly Scores Analysis [48];

- Moving Principal Components Analysis (MPCA) [15]. 


\section{Validation of analysis methods}

\subsection{Numerical Simulation}

To evaluate the efficiency of the proposed algorithms for damage detection, a large dataset from a structure that has been monitored under several levels of damage is required. Since obtaining such datasets from a real structure is difficult, in this study a finite element model of a beam studied by University of Genoa [49] has been used. The main aim of numerical simulation is to simulate the behaviour of a bridge (two-span continuous beam) in healthy and in various damage states [15], see Figure 1. A thermal load has been applied to the beam in order to simulate structural behaviour due to seasonal temperature variations, see Figure 2. Geometric properties of the structure are as follows. Each span is 5 metres in length (L). The beam has a rectangular cross section that is 0.50 metres deep (h) and 0.30 metres wide (t). The beam is discretised into 600 4-node quadrilateral elements, i.e. 100 elements in the x-direction (beam length) and 6 elements in the y-direction. Each element of the mesh has a length of 0.10 meters and a height of 0.08 meters.

In the FE model, both thermal and moving loads can be applied. Simulating damage as stiffness reduction in one or more sections of the beam is also possible. The response is measured by means of a 'virtual' monitoring system installed in the structure. As shown in Figure 1, the monitoring system is composed of six pairs of elongation sensors; six sensors are located on the lower surface and six on the upper surface. Each sensor has a gauge length of 1 meter. The sensors are assumed to have a measurement precision of 2 microns. 
Cracks are simulated in order to model local degradation in the material properties. The structural behaviour is simulated in both healthy and damaged states, in order to test whether the statistical algorithms are able detect when and where damage occurs. Various combinations of damage location and severity are simulated.

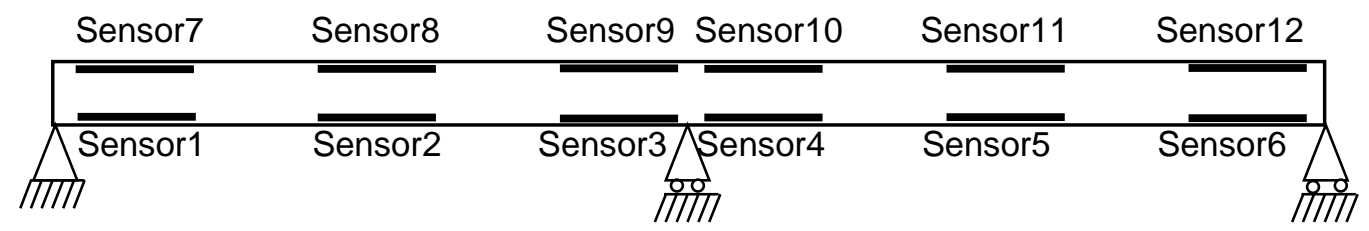

Figure 1: Schema of the FE model with sensor locations used to test the methodology (black lines). In subsequent figures, sensors are referred to as sn1, sn2, etc.

Each simulated time series is representative of structural response measured at a given sensor location over eight years assuming four measurements per day (11000 measurement events). Time series show harmonic variations due to seasonal variations of temperature in healthy and damaged states, see Figure 3. However, no obvious correlation to damage formation is directly detectable from the elongation sensor time histories. A small shift of periodicity is observed for only high degrees of damage.

The algorithms are tested for the following damage scenarios: 
A1-A5 Damage at sensor 2 with a $80 \%$ reduction in stiffness in 4 finite elements.

Five tests are simulated without daily noise in the sensor measurements. The temperature at the bottom of the beam is set to $\mathrm{T}_{\mathrm{B}}=\mathrm{T}_{\text {ref }}+\eta$ and the temperature at the top is kept constant at $\mathrm{T}_{\mathrm{T}}=\mathrm{T}_{\text {ref }}+10\left[{ }^{\circ} \mathrm{C}\right] . \mathrm{T}_{\text {ref }}$ is a sinusoidal function with period of one year and amplitude $10\left[{ }^{\circ} \mathrm{C}\right] . \eta$ represents a normal random variable that is used to simulate variations in daily temperature and traffic. $\eta$. It has a mean $\mu=0$ and a standard deviation $\sigma$ between $0\left[{ }^{\circ} \mathrm{C}\right]$ and $5\left[{ }^{\circ} \mathrm{C}\right]$ depending on the damage scenario as shown below.

$$
\begin{array}{ll}
\text { A1: } & \sigma=0\left[{ }^{\circ} \mathrm{C}\right] \\
\text { A2 : } & \sigma=1.25\left[{ }^{\circ} \mathrm{C}\right] \\
\text { A3 : } & \sigma=2.5^{\circ}[\mathrm{C}] \\
\text { A4 : } & \sigma=3.75\left[{ }^{\circ} \mathrm{C}\right] \\
\text { A5 : } & \sigma=5\left[{ }^{\circ} \mathrm{C}\right]
\end{array}
$$

B1-B10 Damage at sensor 2 with a 80\% reduction in stiffness in 4 finite elements. The temperatures at the top and at the bottom of the beam are $\mathrm{T}_{\mathrm{T}}=\mathrm{T}_{\text {ref }}$ $+10\left[{ }^{\circ} \mathrm{C}\right]+$ noise $\left(\mu=0\right.$ and $\left.\sigma=5\left[{ }^{\circ} \mathrm{C}\right]\right)$ and $\mathrm{T}_{\mathrm{B}}=\mathrm{T}_{\text {ref }}+$ noise, $(\mu=0$ and $\sigma=3$ $\left[{ }^{\circ} \mathrm{C}\right]$ ), respectively (see Figure 2). Ten scenarios are simulated with noise in the sensor measurements ranging from 0 microns in scenario B1 to 20 microns in scenario B10. Damage is introduced in all scenarios after 7000 measurements. 


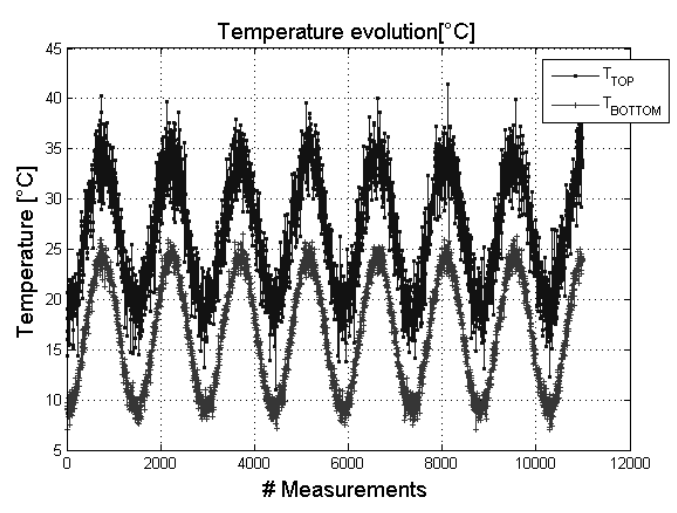

Figure 2: Evolution of the temperature at the top and bottom of the two span continuous beam for a period of 8 years

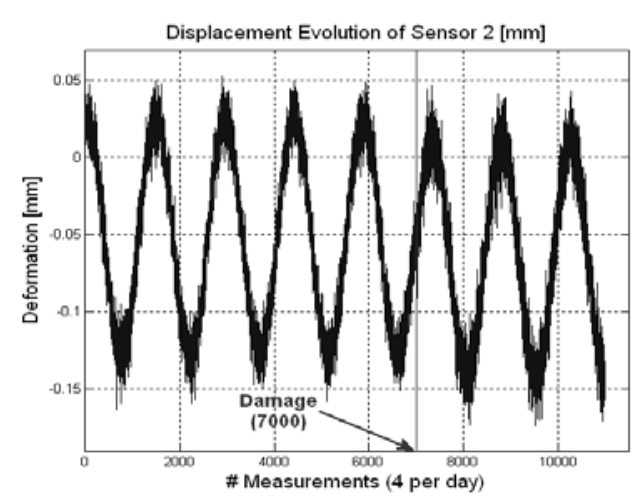

Figure 3: Measurements from sensor 2 taken at a rate of four measurements per day for 8 years. Damage occurs after 7000, from Posenato et al. [15].

\subsection{Damage Detection Methodologies}

Application of the model-free data analysis to Structural Health Monitoring involves an initial phase (called initialization) when the structure is assumed to be undamaged. The aim of this initialization period (training set) is to estimate the variability of the time series and to define the thresholds of the confidence interval $( \pm 3 \sigma, \sigma$ is the standard deviation) for detecting anomalous behaviour. This period is normally one or two years. Once thresholds are fixed, the parameters of the process are monitored to ensure that they are within the predefined range.

In this paper several methods for autoregressive analysis are studied: ARMA, Box-Jenkins and Seasonal ARIMA. The ARMA analysis cannot be applied directly to the time series generated by the sensors due to its seasonal components. The time series are first detrended of the seasonal component. For de-trending the data, three methods are used, (i) 
harmonic regression, (ii) simple average window and (iii) moving average window. To discriminate between damaged and non-damaged states of the structure, a Damage Sensitive Feature (DSF) is defined considering the AR coefficients $a_{i}$ of the ARMA [12]. Thus the proposed DSF for an ARMA model in which the autoregressive part has order $p$ is defined as follows:

$$
D S F=\frac{a_{1}}{\sqrt{\sum_{k=1}^{p} a_{k}^{2}}}
$$

The optimal model order of the ARMA is obtained using the Akaike information criteria (AIC). In order to obtain the AR and MA coefficients the Burg Algorithm is applied. The ARMA coefficients are allowed to vary and are computed after each acquisition based on a moving window composed of only the last 300 measurements. The DSF is then compared with the confidence interval $( \pm 3 \sigma)$ calculated for the training period.

The Box-Jenkins method is applied to the time series from each sensor. The idea is that under normal conditions, the Box-Jenkins representation of time series can be reduced to an ARMA model. When a permanent damage occurs on the structure at time $t$, an exogenous input with the shape of a step function (always 0 before $t$ and 1 after $t$ ) can be introduced into the Box-Jenkins representation. An analysis of the evolution of the coefficient of the exogenous variable (DSF) can identify the occurrence of damage. Before identification of the ARMA for the time series, low frequency components are filtered using wavelet analysis. The estimation of the Box-Jenkins coefficients is done at each step using a Kalman filter on a moving window containing the last 600 measurements. 
The seasonal ARIMA is applied to the time series of each sensor. The coefficients of the ARIMA method can vary with time. They are identified at each step within a moving window that is bigger than the period. Changes in the ARIMA coefficients can reveal damage sustained by the structure. In this study, a window of two years is used and the confidence interval of $\pm 3 \sigma$ is calculated from the variation of the ARIMA coefficients during the third year.

DWT is applied to the time histories of each sensor. Since the recording of strain data is synchronous, a measure of dissociation between data from two sensors gives information about localized changes. Using the definition of a semivariogram [4], the DSF for the DWT can be defined as:

$$
D S F_{i j k}^{X Y}=\frac{1}{2}\left|D_{i j k}^{X}-D_{i j k}^{Y}\right|^{2}
$$

$X$ and $Y$ are the two sensors whose measurements are analyzed. $i$ is the modulation, $j$ is the scale level and $k$ is the translation parameter. If the value of DSF is within the confidence interval of $\pm 3 \sigma$ calculated for the training period, there is no damage. If the DSF is out of $\pm 3 \sigma$ confidence interval, damage is present.

The WPT is not applied directly on the time series from each sensor. Such an approach produces energy plots that are difficult to analyze due to periodic components of the time series. For this reason WPT is applied to the difference between the time series of the two sensors closest to the damage and normalized according to the measurements of the first 
year. The DSF is defined as the energy contained in a node of the decomposition [5-7]. The DSF for the WPT can be defined as:

$$
D S F_{i j}=\sum_{k}\left(D_{i j k}\right)^{2}
$$

Where $i$ is the modulation, $j$ is the scale level and $k$ is the translation parameter and $D_{\mathrm{ijk}}$ is the wavelet coefficient. The WPT is applied along a moving window of constant size. In each iteration, the DSF of all the modulations at scale level 3 is analyzed to evaluate if they are within the $\pm 3 \sigma$ confidence interval calculated during the training period.

The Robust Regression Analysis is applied to the couple of sensors closest to the damage. If the relation between two sensors $y$ and $x$ can be written as $\hat{y}=a x+b$, then at time $t$ a damage is present if for $n$ consecutive times $|y-\hat{y}|>\alpha \sigma . a, b$ are the coefficients of the robust regression line estimated from the measurements of the first year. $\sigma$ is the standard deviation of $y-\hat{y}$ based on the second year. $\alpha$ defines the confidence interval and is set equal to 3 .

For IBM, no moving window is considered. The reference set is composed of all measurements taken during the first year (1460 measurements) and the confidence interval of $\pm 3 \sigma$ is calculated considering the minimum distances of all measurements taken during the subsequent year from the reference set. Damage is detected if a sequence of $N_{a}$ consecutive measurements is outside the confidence interval. 
The Correlation Anomaly Scores Analysis is applied to all time series using a moving window of one year (1460 measurements). At each step a score index $E$ is calculated for all the nodes of the stochastic neighbourhood graph [48]. The confidence interval of $\pm 3 \sigma$ is calculated according the variation of the score $E$ during the second year. Damage is detected if the score of a sensor is out of the confidence interval.

The MPCA is applied to all simulated time histories using a moving window of one year; at each step the eigenvectors related to the main eigenvalues are analyzed. The confidence interval of $\pm 3 \sigma$ is calculated from the variation of the extracted eigenvectors during the second year.

\section{Results}

Results of a comparative study between all the selected algorithms are presented in Table 1. Results show that MPCA and WPT work more effectively than other methods. MPCA is the most appropriate for long-term Structural Health Monitoring tasks as depicted by the simulations in this study. Only MPCA and WPT are able to detect all damage scenarios.

In this section results are shown for three scenarios for each algorithm. The selected scenarios are A1, B1 and B10. The aim is to show the influence of noise on damage detection. A1 and B10 are the two extreme scenarios, i.e., without noise and with maximum noise, while B1 is a scenario with a level of noise that is common in civil engineering. 
In Figure 4-6, autoregressive results are presented for three scenarios. All the autoregressive algorithms detect the damage only in scenario A1. The value of the DSF exceeds the confidence interval immediately after the introduction of the damage. In scenarios B1 and B10, the DSF remains bounded within the confidence interval such that the damage is not detected. The variations due to the added noise mask the damage. When damage is detected, there is no information regarding whether the anomalies are temporary or permanent. The autoregressive analysis performs well only for scenario A1. One reason is that the strategy used to simulate the thermal load is based on a random process such that the thermal load at time $t$ is uncorrelated with the load at time $t-1$. Consequently, a measurement at time $t$ cannot be expressed as a linear combination of the previous measurements. Autoregressive analysis is not appropriate for measurement series in which there is a poor relation between the current measurement and past values.

Discrete wavelet decomposition results in a tree in which each node represents the variation with time of a specific frequency band. Figure 7 is a plot of the damage sensitive feature $D S F_{12}^{23}$ (see Equation 2) that is a result of wavelet decomposition of the difference between measurements from sensors 2 and 3. Only in scenario A1, damage is detected. For the other two scenarios, the DSF remains bounded inside the confidence interval. The damage is not detected since the DWT takes into account only changes of signals over time. These results show that DWT is sensitive to noise. 
Figure 8 shows the $\mathrm{DSF}_{31}$, defined according to Equation 3, from the WPT of the difference between measurements taken from sensors 2 and 3. Damage initiation is detected in all the scenarios. However, results do not indicate if the damage is permanent or temporary. In all scenarios, the value for the DSF exceeds its confidence interval after the damage. In scenario A1, after the damage the DSF remains outside the confidence interval and the damage can be considered to be permanent. In scenarios B1 and B10 the DSF takes values outside of its confidence interval but does not remain permanently outside the thresholds. The algorithm is able to detect the event for scenarios B1 and B10. However, it is not clear from the DSF if the anomaly is temporary or permanent.

In Figure 9, RRA applied to measurements from two sensors that are closest to the damage is shown. The first plot gives the regression line calculated for scenario A1. In this scenario, the shift of the regression line due to the damage is visible and this shift is permanent. The second and the third plots are for scenarios B1 and B10. They show the evolution of the absolute value of the difference between the deformation at sensor 2 and the regression line. In scenario B1 the damage is detected because there are several consecutive points outside the confidence interval after the introduction of the damage. In scenario B10 the damage is not detected because all the points remain bounded inside the confidence interval.

In Figure 10 the results of the IBM applied to the three sensors that are closest to the damage are presented for three scenarios. The reference set is composed of all 
measurements taken in the first year and the confidence interval of $\pm 3 \sigma$ is calculated considering all the minimum distances of measurements taken during the subsequent year from the reference set. For all three scenarios the minimum distance from the training set (reference period) is plotted. In scenario A1, the moment when the damage occurred is detected because after damage, all points are outside the confidence interval. The damage is also detected in scenario B1 as there are many consecutive points outside of the $3 \sigma$ threshold. In scenario B10 damage is not detected as all the points remain inside the confidence interval.

In Figure 11, the Correlation Anomaly Scores Analysis calculated for the K-Nearest Neighbours $(K=3)$ of the sensor closest to the damage are reported for three scenarios. The damage initiation and the fact that it is permanent, is detected only in scenarios A1 and B1. The damage is detected because the $E$ score coefficient calculated for Sensor 2 is permanently outside the confidence interval. In scenario B10 the damage is not detected because the $E$ score coefficient remains bounded inside its confidence interval.

In Figure 12, MPCA diagnostic plots of the eigenvectors related to the main eigenvalues are shown for three scenarios. Damage initiation and its location are detectable in all graphs by following the evolution of the two main eigenvectors. Specifically, one of the eigenvectors (eigenvector 11) indicates a new state, while the other (eigenvector 12) indicates when the damage has occurred. In the MPCA the location of damage can be inferred from the main eigenvectors because the sensors close to the damage have one or more rapidly changing components. In all figures, the evolution of main eigenvectors of the 
sensor closest to the damage are shown. The MPCA has the advantage of discovering the time of damage occurrence from the main eigenvectors, and also the damage location by observing the components with higher variation. It can also indicate whether or not the structure reaches a new stable condition.

\section{Scenario A1}

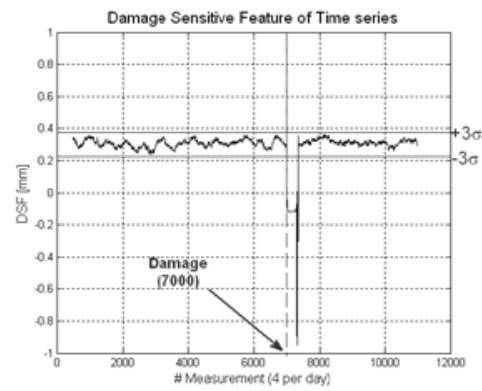

\section{Scenario B1}

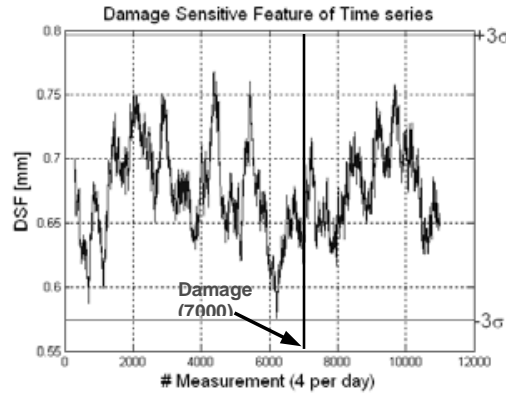

\section{Scenario B10}

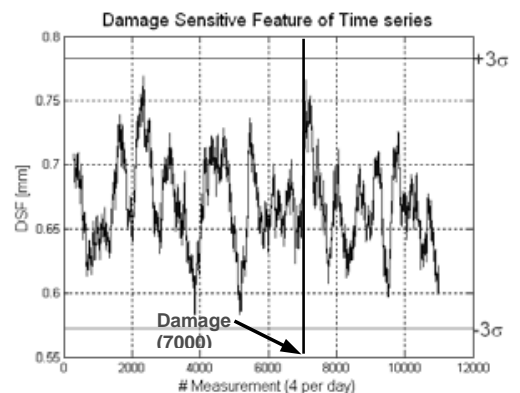

Figure 4: Damage Sensitive Feature (DSF) calculated using ARMA analysis for the sensor closest to the damage for scenarios A1, B1 and B10. Only damage in scenario A1 is detected. The confidence interval of $\pm 3 \sigma$ is calculated according the variation of the DSF during the first year (x-axis: number of measurements; y-axis: DSF).

Scenario A1

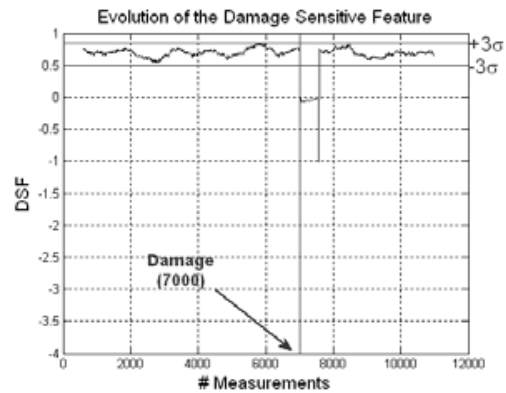

Scenario B1

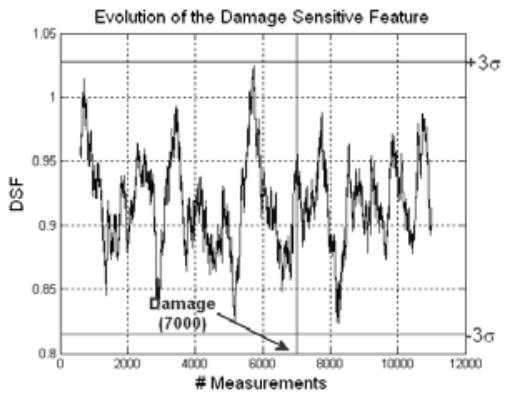

Scenario B10

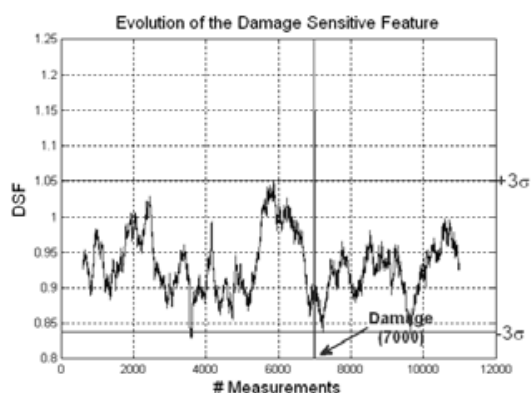

Figure 5: DSF evolution from Box-Jenkins analysis of measurements from sensor closest to the damage. Only damage in scenario A1 is detected. The confidence interval is calculated according the variation of the DSF during the training period (first year) (x-axis: number of measurements; y-axis: DSF). 
Scenario A1

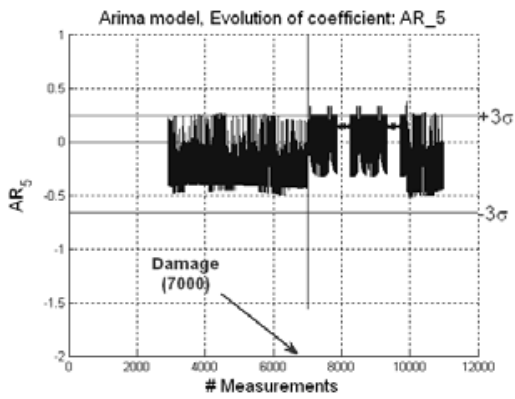

Scenario B1

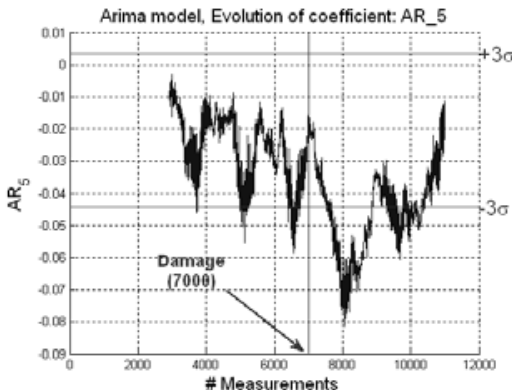

Scenario B10

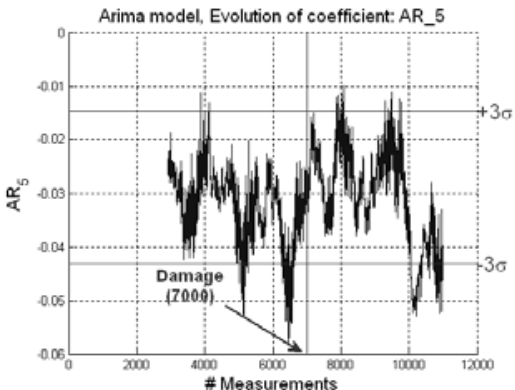

Figure 6: Plots of one coefficient of the ARIMA method calculated for the sensor closest to the damage. Only damage in scenario A1 is detected. The confidence interval is calculated the variation of the coefficients during the third year (x-axis: number of measurements; $y$-axis: evolution of an auto-regressive coefficient).

\section{Scenario A1}

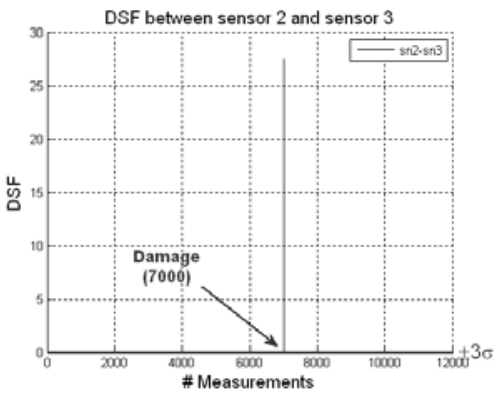

\section{Scenario B1}

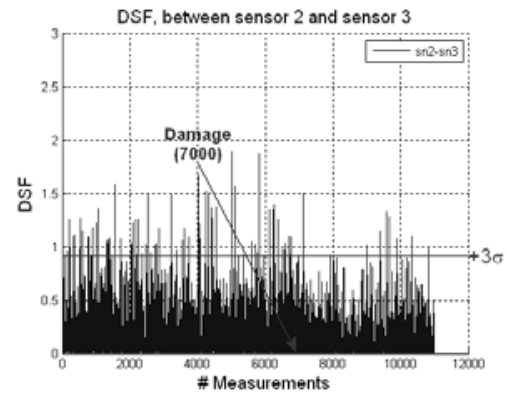

\section{Scenario B10}

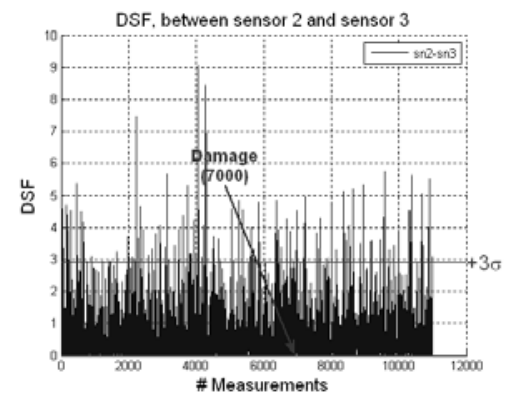

Figure 7: DSF from the DWT of the node [1,2] calculated for the difference between sensors 2 and 3. Only damage in scenario A1 is detected. The training set for the definition of the confidence interval contains all the measurements done in the first year (x-axis: number of measurements; y-axis: DSF). 
Scenario A1

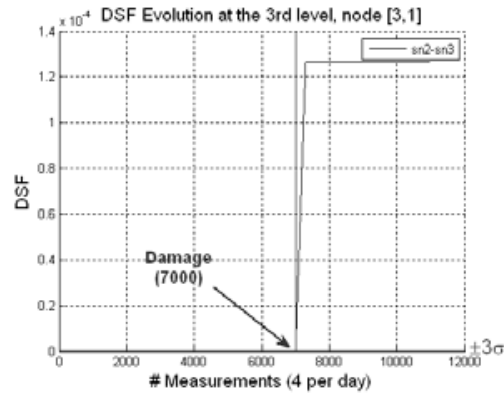

Scenario B1

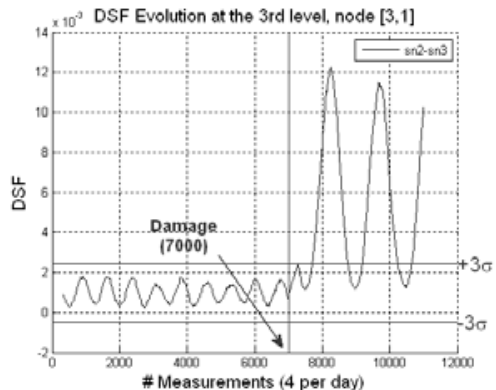

Scenario B10

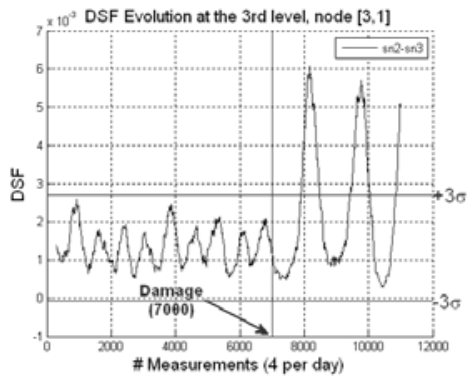

Figure 8: DSF from the WPT of the node [3,1] calculated for the difference between sensors 2 and 3 . The damage initiation is detected in all three scenarios. The training set for the definition of the confidence interval contains all the measurements done in the first year (x-axis: number of measurements; y-axis: DSF).

Scenario A1

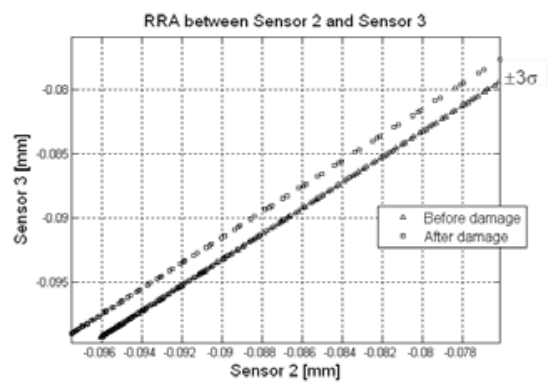

Scenario B1

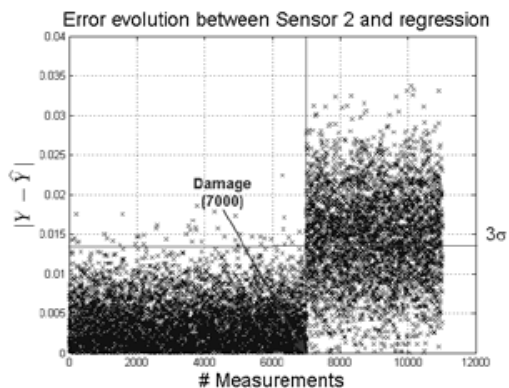

Scenario B10

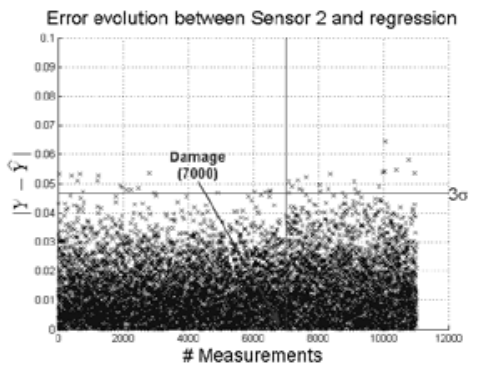

Figure 9: Robust Regression Analysis method for measurements from the two sensors that are closest to the damage. In the first plot the regression line shifts due to the damage (x-axis: deformation from sensor 2 in millimeters; $y$-axis: deformation from sensor 3 in millimetres). The second and the third plots show the absolute value of the error evolution between the deformation at sensor 2 and the regression line (x-axis: number of measurements; $y$-axis: error in millimetres). The confidence interval is calculated using the measurements of the first year. 
Scenario A1

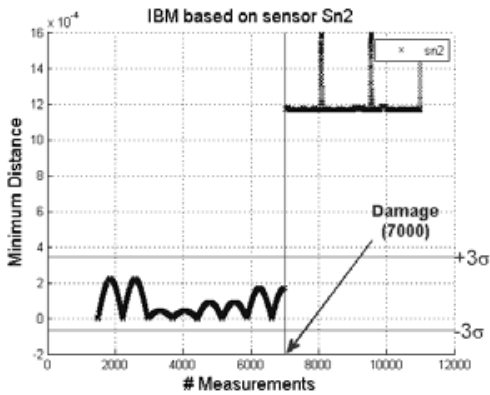

Scenario B1

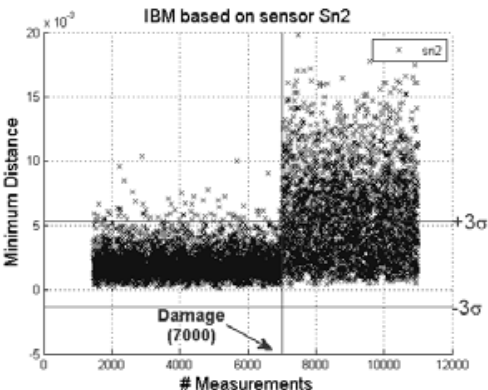

Scenario B10

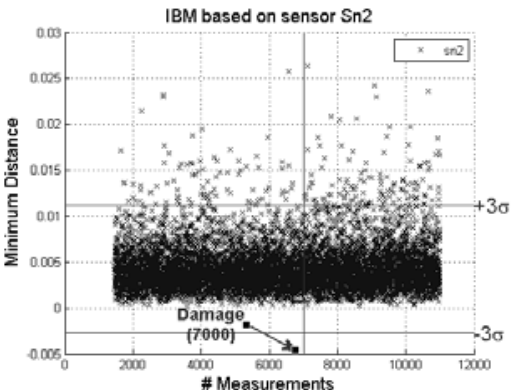

Figure 10: Instance-based method calculated for the three sensors that are closest to the damage. They show that damage initiation and that the situation is permanent and not temporary. (x-axis: number of measurements; $y$-axis: distance in millimetres). Damage is detected in scenarios A1 and B1.

\section{Scenario A1}

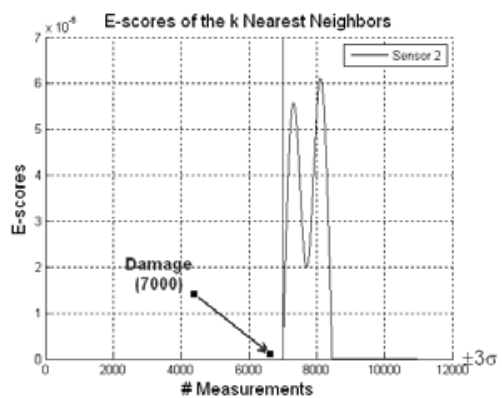

\section{Scenario B1}

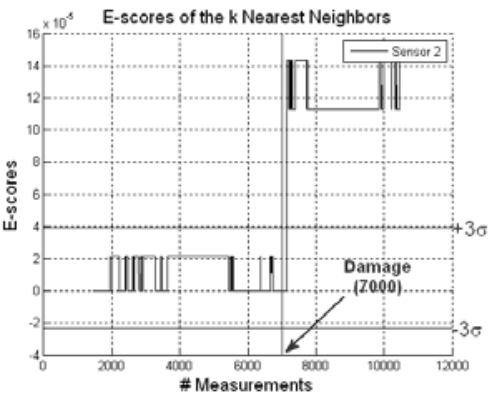

\section{Scenario B10}

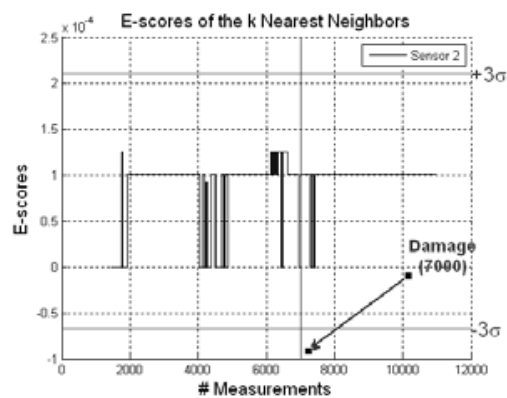

Figure 11 Plots of Correlation Anomaly Scores Analysis calculated for the K-Nearest Neighbours (K=3) closest to sensor 2. They show the damage initiation and that the situation is permanent and not temporary (x-axis: number of measurements; y-axis: correlation anomaly scores $E$ ). Damage is detected in scenarios A1 and B1.

\section{Scenario A1}

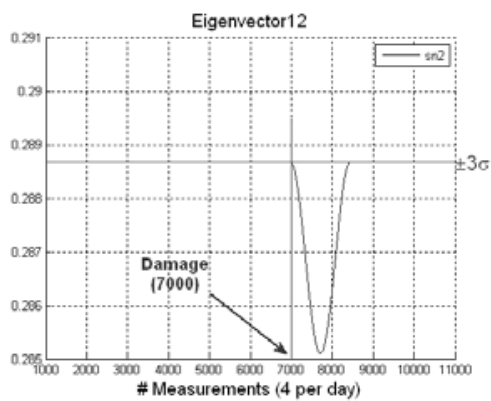

\section{Scenario B1}

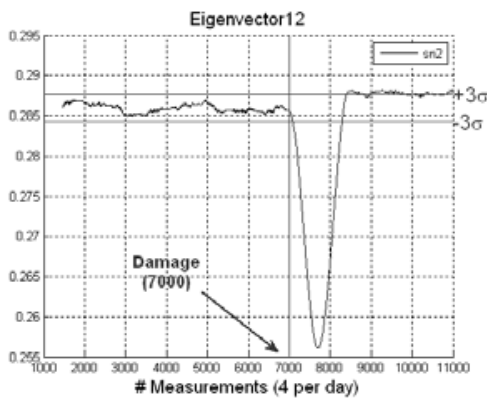

\section{Scenario B10}

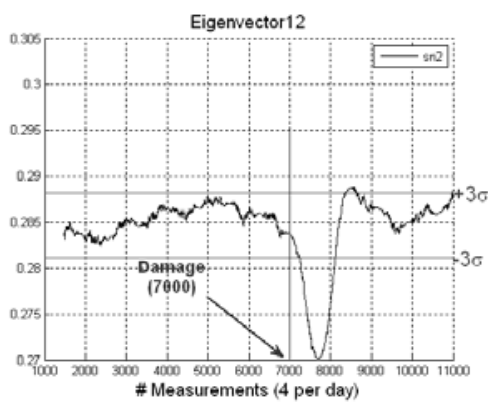



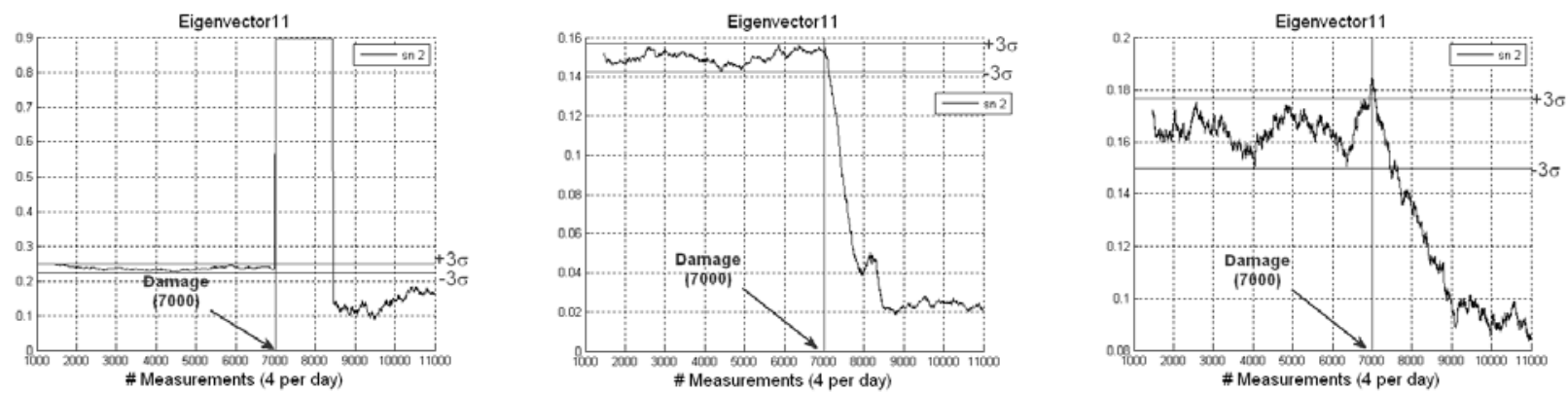

Figure 12: Plots of the eigenvectors related to the two main eigenvalues calculated using MPCA. They detect the damage and identify its location. Eigenvector 11 identifies the new state of the structure when it becomes stable again. Eigenvector 12 detects the damage introduced after 1750 days (measurement 7000) (x-axis: number of measurements; y-axis: eigenvector). The damage is detected in all three scenarios.

Figure 5 - 12 and Table 1 illustrate that noise reduces the ability to detect damage. In Figure 13 the influence of the noise on damage detection is shown for the case studied in this paper. Few algorithms are able to discover damage from time histories with levels of noise commonly observed in civil engineering applications (shown by dashed line in Figure 13).

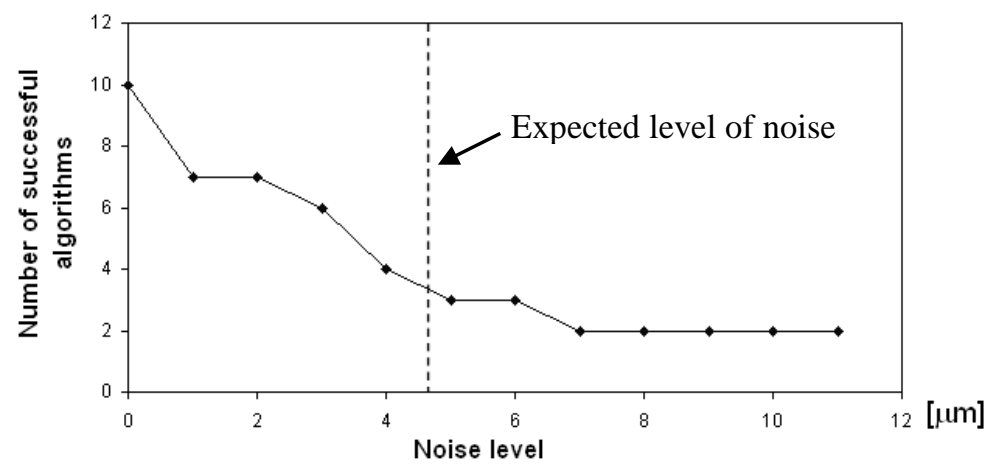

Figure 13: Plot showing the number of algorithms that are able to detect damage for increasing levels of noise in measurements. Results are for a damage scenario at sensor 2 with $80 \%$ reduction in stiffness in 4 cells

Table 1 and Figure 13 indicate that the number of algorithms that are able to detect damage decreases with increasing levels of noise in the measurements. Only two algorithms, MPCA and WPT, are able to detect the damage in all simulated scenarios. The MPCA has 
an advantage over WPT in that it is able to differentiate between events that introduce a permanent change in structural behaviour (see Figure 12) and those that temporarily alter structural behaviour. In the following sections, methods for data preparation are studied in order to ensure that MPCA is effective in the presence of outliers and missing data.

\section{Extending MPCA to accommodate missing data and outliers}

Currently, many software packages are available for data analysis. From the perspective of practical data analysis, a limitation in several of these software packages is that they cannot accommodate missing data other than by means of list-wise deletion. In addition, many of them do not take into account the presence of outliers, which may also distort the results of the analysis and thus affect damage detection. Figure 14 and 15 show, respectively, situations of missing data and the presence of outliers. The purpose of this section is to describe and evaluate simple and practical solutions for missing data and outliers. Figure 16 presents the sequence of steps necessary to extend MPCA for data interpretation tasks in structural health monitoring. Clustering is necessary for the analysis of datasets from monitoring situations that involve a large number of sensors, probably of various types. Each of the steps in Figure 16 is discussed in detail in the following subsections. 


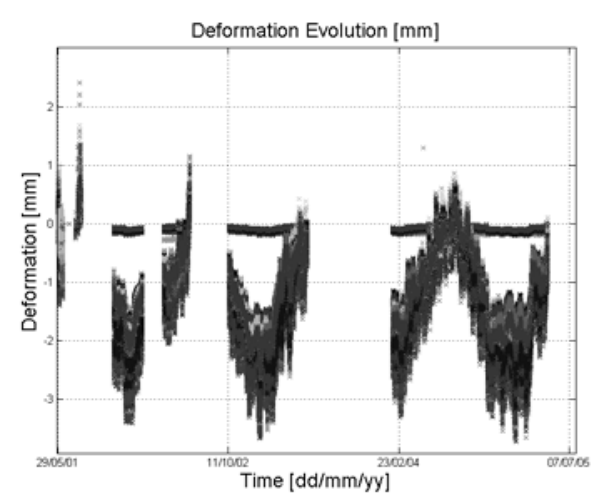

Figure 14: Missing data completely at random in data obtained from the Colle Isarco Bridge on the Brennero Highway in Italy.

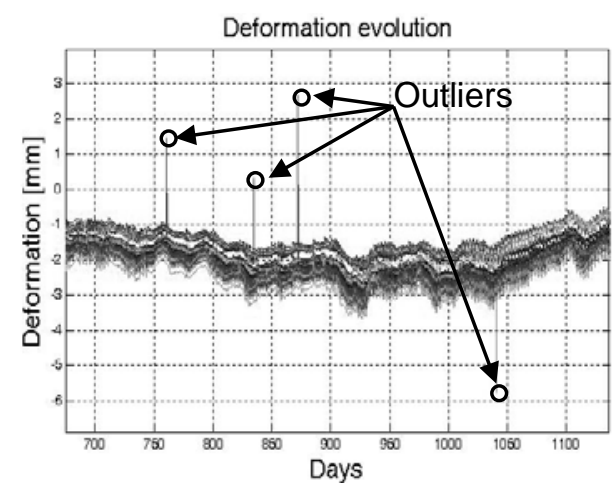

Figure 15: Outliers in data obtained from the Colle Isarco Bridge on the Brennero Highway in Italy.

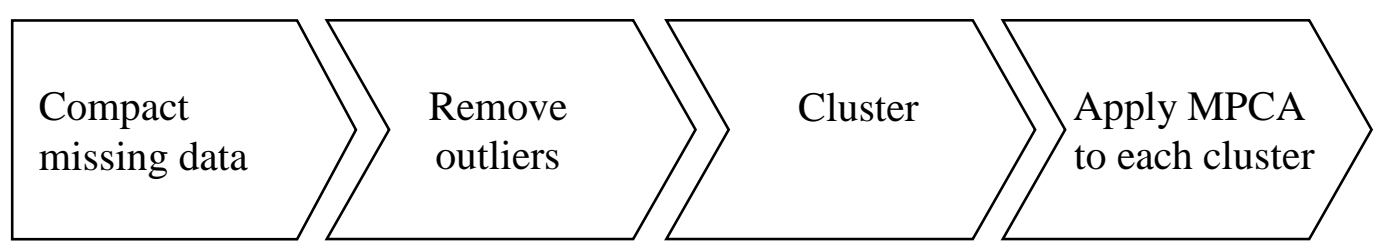

Figure 16: Sequence of steps in data interpretation

\subsection{Missing data}

Measurement data sets that have missing data are common. Missing data can occur for reasons such as: power supply interruptions, structural maintenance, revision of reading units or other unpredictable situations. Most algorithms are unable to manage periods where measurements from sensors are missing. Replacement of incomplete data may add more uncertainty and increase the difficulty of damage detection. MPCA can successfully manage situations of missing individual measurements and missing complete sets of measurements at random. This section describes the methodology. 
Datasets from monitoring systems can be expressed as matrices that contain the history of measured parameters:

$$
\mathbf{M}(t)=\left[\begin{array}{cccc}
u_{1}\left(t_{1}\right) & u_{2}\left(t_{1}\right) & \cdots & u_{N s}\left(t_{1}\right) \\
u_{1}\left(t_{2}\right) & u_{2}\left(t_{2}\right) & \cdots & u_{N s}\left(t_{2}\right) \\
\cdots & \cdots & \cdots & \cdots \\
u_{1}\left(t_{N}\right) & u_{2}\left(t_{N}\right) & & u_{N s}\left(t_{N}\right)
\end{array}\right]
$$

$N$ is the total number of observations during the monitoring period, and $N_{s}$ is the total number of sensors in the monitoring system. A moving window composed of $N_{w}$ observations is used. The analysis employs a matrix $\mathbf{U}\left(N_{w}, N_{s}\right)$ that contains a fixed number of rows of $\mathbf{M}$. The main concept of the MPCA is that $\mathbf{U}(\mathrm{t})$ can be split into a mean part $\overline{\mathbf{U}}$ and a second part $\boldsymbol{\eta}(\mathrm{t})$ with zero-time average such that $\mathbf{U}(\mathrm{t}), \overline{\mathbf{U}}, \boldsymbol{\eta}(\mathrm{t}) \in \mathbf{R}^{\mathbf{N}_{\mathbf{w}} \mathbf{x} \mathbf{N}_{\mathrm{s}}}$. The time series $\mathbf{U}(\mathrm{t})$ can be written as:

$$
\mathbf{U}(\mathrm{t})=\overline{\mathbf{U}}+\boldsymbol{\eta}(t)
$$

Using the linear projection of the principal component analysis computed on the covariance matrix of $\mathbf{U}$, Equation (4) can be written as:

$$
\mathbf{U}(\mathrm{t})=\overline{\mathbf{U}}+\sum_{i=1}^{N s} a_{i}(t) \boldsymbol{\psi}_{i}
$$

$a_{i}(\mathrm{t})$ is a vector $=\left[a_{i 1} \ldots a_{i N w}\right]^{\prime}$ and it is time dependent and $\psi_{i}$ is position dependent. Each vector $a_{i}(\mathrm{t})$ describes the principal trend of the system; if the sensors are correlated then at time $\mathrm{k} a_{i k}$ (referred to the main eigenvectors) describes the ith-trend which is, common to all sensors. The eigenvector $\psi_{i}=\left|\psi_{\mathrm{i} 1} \psi_{\mathrm{i} 2} \ldots \psi_{\mathrm{i} 1}\right|$ ' represents the contribution of $a_{i k}$ to each sensor. 
In case of missing a single measurement at random the covariance of the measurement series with missing data is calculated using fewer values and the accuracy depends on the number of missing values in relation to the dimension of the moving window $N_{w}$. The situations of missing complete measurements are less critical since the dataset can be compacted by removing all the rows in which no measurements are present, see Figure 17. The effect of compaction is visible only in $a_{i}(\mathrm{t})$. The main $\psi_{i}$ remain constant (if no significant damage occurred in the period during which there are no measurements, otherwise changes will be visible in the components of $\psi_{i}$ ).

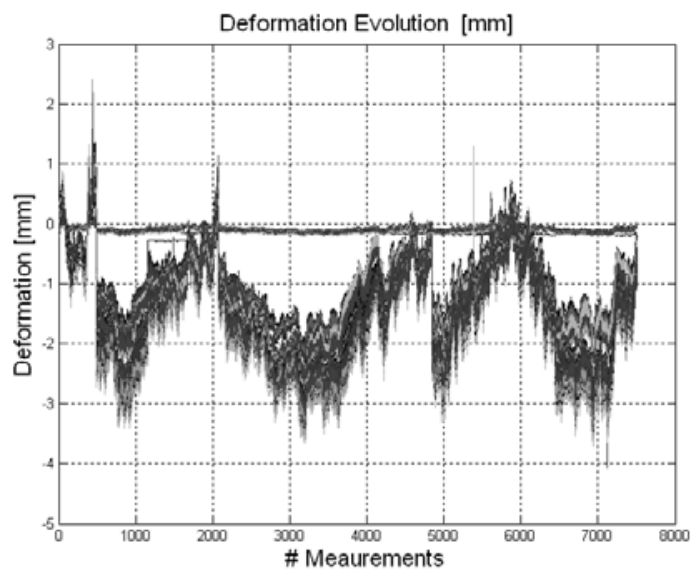

Figure 17: Time series after compacting measurement series with missing data (MCAR) (shown in Figure 14) obtained from the Colle Isarco Bridge on the Brennero Highway in Italy

Thus MPCA manages situations of missing complete measurements and missing a single measurement at random with little pre-processing of the data. For the first case the data set is compacted removing rows without measurements. For the second case, covariances between sensors are calculated only when measurements from both sensors are available. 


\subsection{Outliers}

Outlier data points are often present in measurement databases that are obtained from structural monitoring systems. Their presence can decrease the sensitivity and the ability to determine the presence of damage in structures. In Figure 15, a simulated time history with $1 \%$ of outliers is shown.

In order to study the effect of outliers on the performance of signal processing algorithms, a series of numerical experiments are conducted. Outliers are randomly added to the measurements simulated using damage scenario B1. The number of outlier data points added to each sensor is $1 \%$ of the total data points. Figure 3 shows time series with and without outliers. The results from the MPCA analysis are presented in Figure 19. In the presence of outliers, the MPCA is unable to detect damage.

Figure 20 shows the influence of the number of outliers on damage detection. Only one algorithm - RRA, is able to discover damage in the presence of outliers as expected in civil engineering applications (shown using dotted line in Figure 20). A small number of wrong measurements may significantly reduce the damage detection capabilities of most algorithms. For successful application of other techniques including MPCA, data must first be treated to remove outliers. 


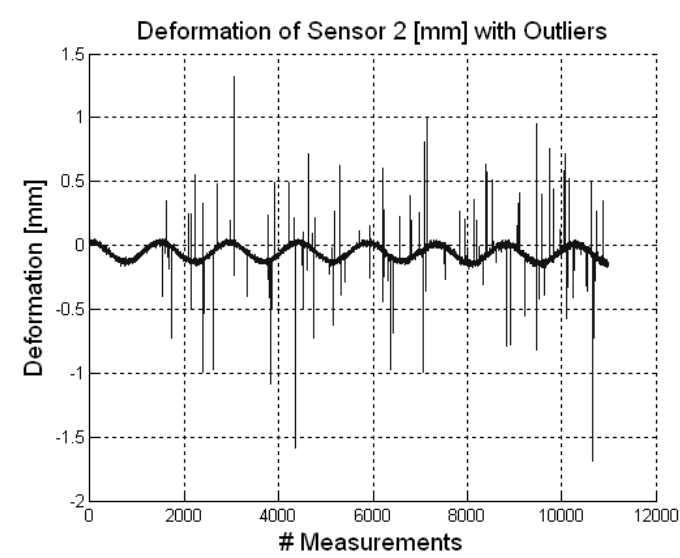

Figure 18: Deformation at sensor 2 in scenario B1 when $1 \%$ of outliers are introduced.

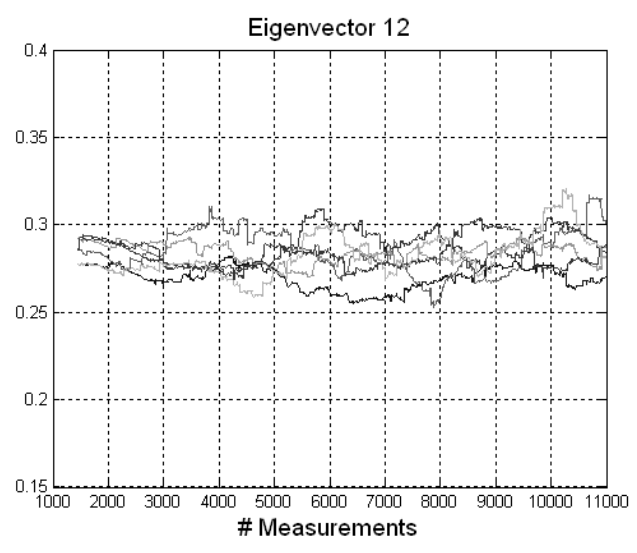

Figure 19: Eigenvector corresponding to the main eigenvalue evaluated by MPCA for scenario B1 with artificial outliers. The algorithm fails to detect damage.

Methodologies exist to find outliers and replace them with appropriate values. Three algorithms are compared for the detection and cleaning of outliers.

Three- $\sigma$ analysis: This algorithm computes the average $\mu$ and the standard deviation $\sigma$ for all the measurements in the period between $t$ and $t-N_{p}\left(N_{p}\right.$ is a small window in which the measurements from the sensors are supposed to be stationary) for each sensor $s_{i}$. If an outlier is detected at time $t+1$,i.e., $s_{i}(t+1)$ is out of the bound $\mu \pm 3 \sigma$, then it is replaced with the value of the median.

Auto-regressive analysis: For each sensor $s_{i}$ at time $t$, an auto-regressive model is estimated considering only the last $N_{p}$ measurements. The standard deviation $\sigma_{r e f}$ calculated for the entire reference period defines the threshold used for outlier detection. At time $t$ an outlier is detected if $\left|s_{i}(t)-\hat{s}_{i}(t)\right|>3 \sigma_{\text {ref }}$. The outliers are then replaced with the value $\hat{s}_{i}$ obtained from the auto-regressive model. 


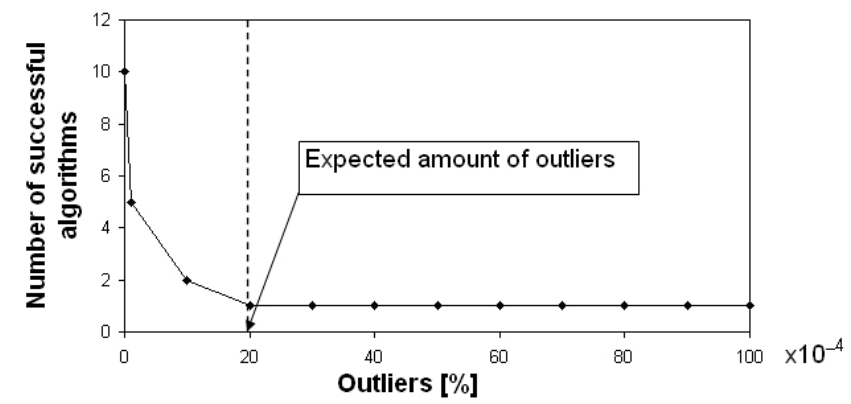

Figure 20: Plot showing the number of algorithms that are able to detect damage for increasing numbers of outliers in measurement sets from damage scenario in which 4 cells at sensor 2 have a stiffness reduction of $80 \%$.

Interquartile Range Analysis (IQR): This algorithm is described below.

Input $\quad-\quad$ A data set $S$ composed of all measurements $\left(N_{m}\right)$ of the sensors $\left(N_{s}\right)$

- $\quad N_{p}$ Number of points in the moving window

- Allsensors = list of all available sensors

Output $\quad$ A data set $S_{\mathrm{c}}$ composed of all measurements $\left(N_{m}\right)$ of the sensors $\left(N_{s}\right)$

without outliers

Algorithm:

$\mathrm{S}_{\mathrm{c}}=S$

For each sensor $s_{j}$ in Allsensors

For $p=1$ to $N_{m}-N_{p}$

$M=$ list of measurements of $s_{j}$ from $p$ to $p+N_{p}$

$M_{s}=$ sort $M$ in ascending order 


$$
\begin{aligned}
& Q_{1}=\text { value of } M_{s} \text { at position } 1 / 4 \text { of } N_{p} \\
& Q_{3}=\text { value of } M_{s} \text { at position } 3 / 4 \text { of } N_{p} \\
& \text { Median= value of } M_{s} \text { at position } 1 / 2 \text { of } N_{p} \\
& \text { LowerThreshold }=Q_{1}-1.5\left(Q_{3}-Q_{1}\right) \\
& \text { UpperThreshold }=Q_{3}+1.5\left(Q_{3}-Q_{1}\right) \\
& \text { if value of } M \text { at position } 1 / 2 \text { of } N_{p}>\text { UpperThreshold or } \\
& \text { value of } M \text { at position } 1 / 2 \text { of } N_{p}<\text { LowerThreshold } \\
& \text { then an outlier is detected }
\end{aligned}
$$$$
S_{c}\left(S_{j, p}+1 / 2 * N_{p}\right)=\text { Median }
$$

Next $p$

Next $s_{j}$

All three algorithms are able to detect and remove outliers; however, IQR has certain superior characteristics. Three- $\sigma$ algorithm is influenced by the number of outliers present inside the moving window during the analysis. It works properly only when there are no outliers in the moving window otherwise the standard deviation is biased by these values and consequently points that are outliers may be classified as good. The auto-regressive analysis requires a certain relation between the data and its past values. Such a relationship is not always present. Furthermore, the estimated autoregressive model may be unstable. Hence replacing outliers with the values suggested by the model can lead to a divergent time series which, potentially, tends to infinity. 
The IQR algorithm is based on robust analysis and therefore is applicable even when there are several outliers in the analyzed datasets. For IQR to be effective, in each iteration only the central value in the window is compared against the thresholds. Otherwise there is a risk of classifying measurements due to changes in structural behaviour as outliers. Thus a new measurement $m$ is not immediately checked to be an outlier. An additional number of measurements is required such that $m$ becomes the central value in latest moving window.

Consider a case in which the dimension of the window for outlier detection is $N_{p}$ and a permanent damage occurs near sensor $s_{i}$. The damage introduces a deviation in the time series starting from the $k$-th measurement; $s_{i}(k)$ is the first measurement after damage. Suppose that IQR performs the outlier check on the last measurement in each moving window. Then the measurement $s_{i}(k)$ could be classified as an outlier since it is further from the rest of the measurements $M$. Therefore it would be replaced with the median. Consequently all the measurements taken after the occurrence of damage would also be classified as outliers and replaced with the median. The abrupt permanent change will not be detected by the anomaly detection algorithm. Therefore, there is a risk of classifying measurements that reflect an abrupt permanent change in system behavior as outliers. This risk is reduced significantly if the IQR algorithm checks only the central measurement in the moving window. Therefore, in the event of damage, there is at least $N_{p} / 2$ measurements after the permanent change. Consequently the measurements indicating damage will not be classified as outliers. 
Figure 21 shows the time series of sensor 2 from Scenario B1 after the cleaning of outliers with IQR, while Figure 22 and 23 show the MPCA results applied to this cleaned dataset for identifying the damage.

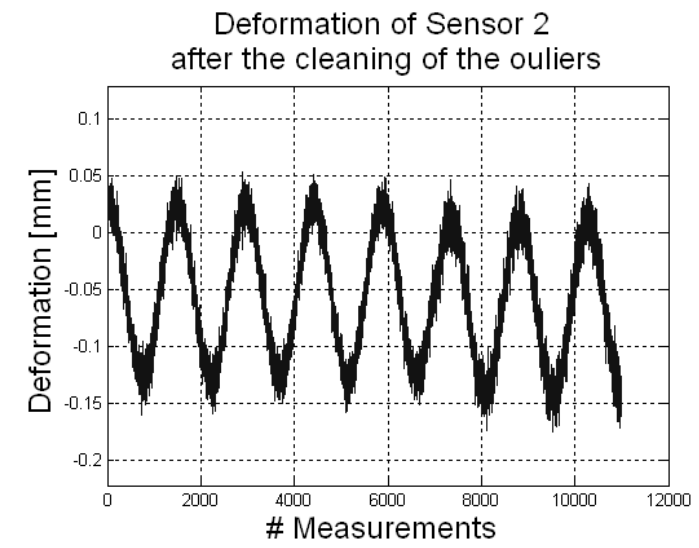

Figure 21: Measurement series from sensor 2 in scenario B1 after the removal of outliers using the IQR algorithm.

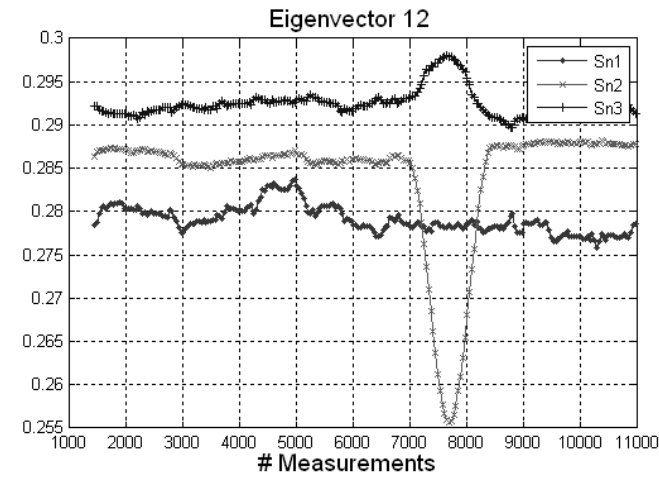

Figure 22: Eigenvector corresponding to the main eigenvalue calculated with the MPCA in scenario B1 after the outliers are removed using the IQR.

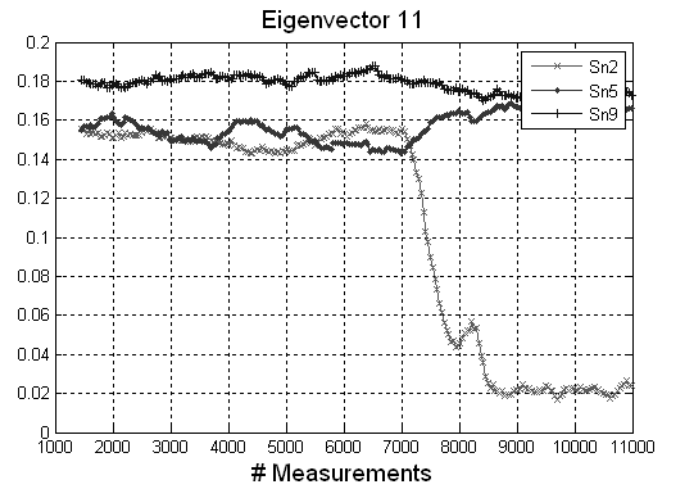

Figure 23: Eigenvector corresponding to the second eigenvalue calculated with the MPCA in scenario B1 after the outliers are removed using the IQR.

Results (Figure 22) show that IQR is a simple and robust cleaning procedure to remove outliers from datasets. This procedure is applied to the time series generated by each sensor 
before analyzing for damage detection. Results show that after the filtering of outliers using the IQR algorithm, the damage detection becomes once again feasible.

\subsection{Clustering}

In applications such as the Götaälvbron bridge in Sweden, where more than 70000 sensors are installed in a single bridge, the new I35 bridge in Minneapolis with more than 350 sensors and the Nam Ngum II dam in Laos with more than 250 measurement instruments, data interpretation can be a very complex task due to the large amount of sensors. Most algorithms cannot process measurements from many sensors at the same time. The MPCA will also fail in these conditions for two main reasons: the time spent for the processing and for the interpretation of the results. The computation involved in finding and inverting a covariance matrix of large dimensions is time-consuming. Moreover, the analysis is not useful when sensors of the same type are installed in uncorrelated positions or when measurements of different types are analyzed.

Organizing the data in clusters is also useful when sensors stop working. In the case when one (or more) sensor stops functioning, the covariance matrix becomes singular and it is not possible to calculate eigenvalues and the eigenvectors. In such circumstances, the only available solution is to remove the initial data associated with the failed sensors from the calculation and to recalculate from the beginning of the monitoring (even the $\pm 3 \sigma$ thresholds have to be recalculated). When clustering is used, only the clusters that contain data from failed sensors have to be recalculated. Another drawback of the analyzing all the sensors together is that if several sensors with different trends are considered there will be 
more variability in the eigenvalues and eigenvectors, This introduces more unevenness and affects the $\pm 3 \sigma$ thresholds used for damage detection.

MPCA works well when sensor data show the same trend; the type of sensor is not important as long as measurements are strongly-correlated. The principle of the MPCA methodology is to limit the analysis to eigenvectors that correspond to the main eigenvalues; when uncorrelated sensor data are analyzed, additional eigenvalues and eigenvectors reduce reliability. On the contrary, if the sensors are highly correlated, there will be only few relevant eigenvalues and the data analysis is more likely to detect damage.

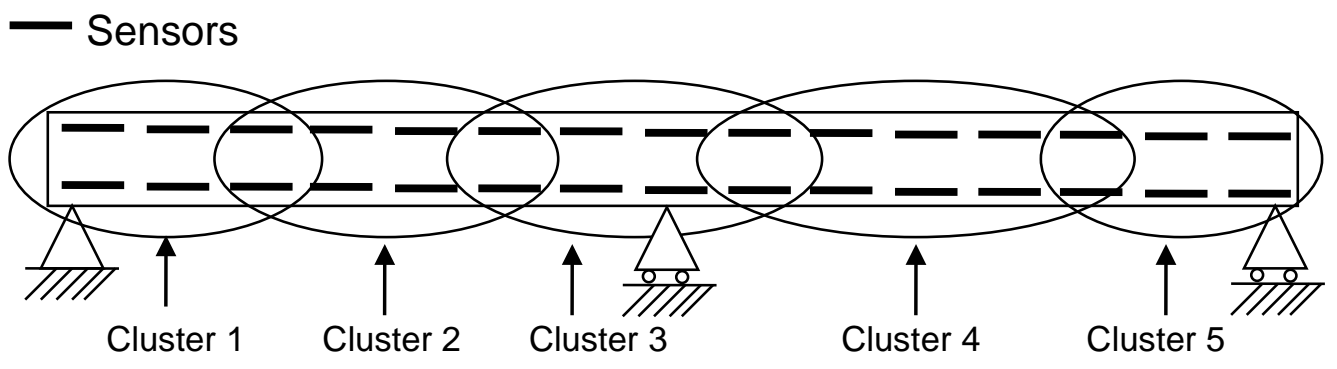

Figure 24: Example of an overlapping clustering created according correlations between sensors.

Figure 24 shows an example of clustering with overlapping. The purpose of the clustering algorithm is to extend MPCA to applications where a significant number of sensors are installed on the structure. The aim of this algorithm is to cluster and gather sensors with comparable trends and similar sensitivities to damage. MPCA is then applied to each cluster of sensors. The clustering overlapping algorithm based on correlations between sensors is given below. 
Input $\quad-\quad$ A data set $S$ composed of all measurements $\left(N_{m}\right)$ from sensors $\left(N_{s}\right)$

- $\quad K_{c}$ threshold for the creation of the clusters

- $\quad N_{\text {ref }}$ Number of points in the reference period

- Allsensors = list of all available sensors

Output $\quad-\quad L C$ list of all the clusters

\section{Algorithm:}

1. $L C=\{\}$

2. $\quad \boldsymbol{C}\left(N_{s}, N_{s}\right)=$ correlation matrix calculated on the matrix $\boldsymbol{S}\left(N_{\text {Ref }}, N_{s}\right)$

3. Select the first available sensor $\mathrm{s}_{j}$ inside AllSensors

4. Form a cluster $C_{i}$ that is composed of all sensors having a high correlation with $\mathrm{s}_{j}$, i.e., all sensors corresponding to elements in row $j$ of $C$ with values $>K_{c}$

5. $\quad L C=L C \cup\left\{C_{i}\right\}$

6. $\quad$ Allsensors $=$ Allsensor $-\left\{C_{i}\right\}$

7. While Allsensors $\neq\{\}$ goto step 3

The clustering algorithm groups together the sensors having a correlation higher than a fixed threshold $K_{c}$; the analysis of small groups of sensors obtained after clustering is easy and fast. The overlapping between the clusters is important because without overlapping, damage that is located between two uncorrelated clusters could remain undetected. At the end of the clustering process, each cluster will contain sensors that are spatially close 
(because correlation improves with proximity between sensors) or sensors that are at locations with the same pattern such as those located at symmetrical locations.

The choice of $K_{c}$ influences the number of clusters and the relation between the sensors in each cluster. The value for the constant $K_{c}$ is chosen on the basis of the following considerations:

0 to 0.2 Very weak to negligible correlation

0.2 to 0.4 Weak, low correlation (not very significant)

0.4 to $0.7 \quad$ Moderate correlation

0.7 to 0.9 Strong, high correlation

0.9 to $1.0 \quad$ Very strong correlation

If the value of $K_{c}$ is too low there will be few clusters with many sensors. In this situation it will be hard to detect damage since it will be hidden by low sensor correlations. A useful estimate of the value of $K_{c}$ is the square of the correlation coefficient (i.e., calculate $r^{2}$ ) between sensors. This is an estimate of the amount of variation in the parameter measured by sensor $s_{\mathrm{i}}$ which is directly attributable to the parameter measured by sensor $s_{j}$. A high value indicates that one sensor has a direct and important effect upon another. When there is a high dependency between all the sensors, as in the computer simulation reported in this paper, the damage initiation will be immediately detected by observing changes in the correlations between sensors that are close to the damage and those that are not. 


\section{Full-Scale Structural Health Monitoring}

In this section two full-scale structural applications are presented. Statistical evaluations of structural behavior using MPCA and RRA are presented. The Ricciolo ("curl”) viaduct was built in 2004-2005 at the Lugano North exit of Swiss motorway A2 [50]. It consists of five spans with a total length of 134 meters. The 35-meters long main span crosses Vedeggio torrential river. It is a curved box girder that is post-tensioned in various directions. A view of the completed main span of the Ricciolo viaduct is given in Figure 25 and the crosssection of box girder is shown in Figure 26.

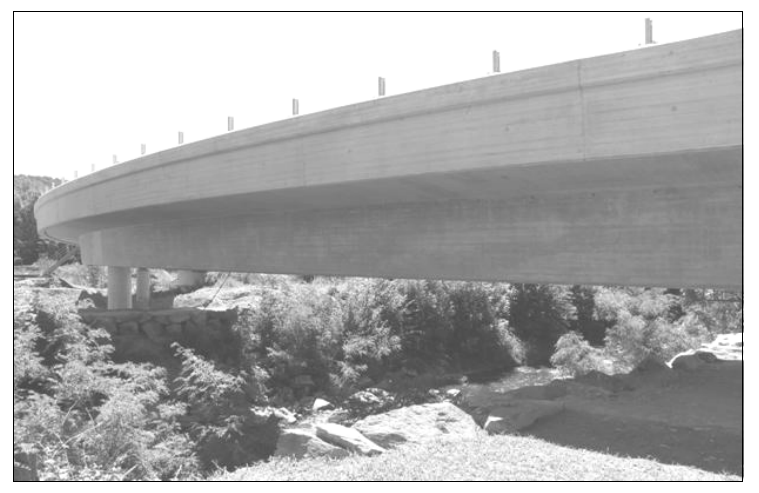

Figure 25: View of the main span of Ricciolo viaduct.

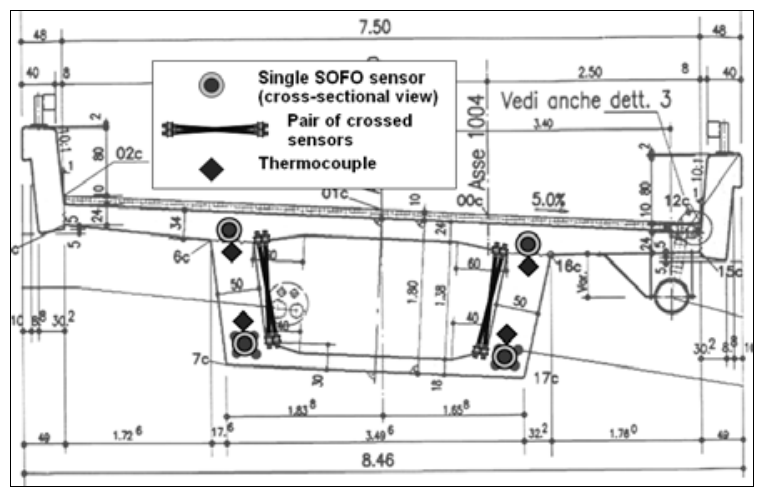

Figure 26: View of the viaduct's cross-section equipped.

Parallel sensors and thermocouples were embedded in the girder by installing them on the rebar cage before pouring of concrete. Crossed sensors and inclinometers were mounted on the hardened concrete surface. The positions of the sensors along the bridge are given in Figure 27. 


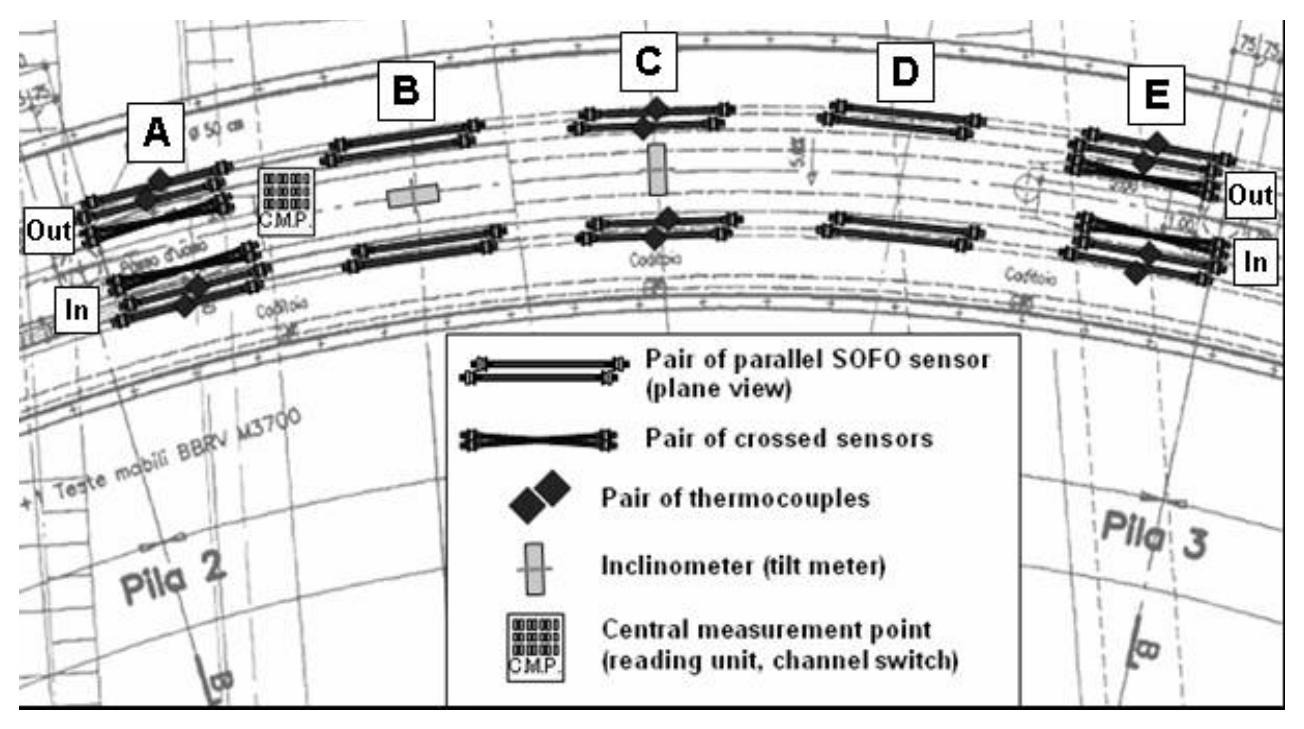

Figure 27: Schematic representation of sensor network on Ricciolo viaduct, plane view, sensors not to scale.

Continuous monitoring started in January 2005 at a rate of one measurement session every hour. This schedule was perturbed a few times in order to use the instruments in other projects. A plot of the average strain is shown in Figure 28; in this graph two sections of missing data are also visible. During the first four and a half months, the bridge was under construction. Important stages having an influence on the stress and strain distributions are given below:

Work schedule from January 10 to May 26, 2005

1. Post-tensioning from 30 to $70 \%$

2. Partial lowering formworks

3. Construction of lateral protection walls

4. Post-tensioning from 70 to $100 \%$
January 12-14

January 17

January 17- April 22

April 25-26 

5. Cast of left side wing
April 25-27
6. Removal of external formworks
April 25-27

In order to apply MPCA and Robust Regression Analysis and to present some examples of results, the data collected after work stages were completed is used as a reference. Since the bridge is in good health condition after the completion of these stages, there are no damage events that could have generated unusual behaviour. For the purposes of demonstration, it is decided to invert the time scale, so that construction work are placed in the "future," and thus appearing as unusual loading. Main eigenvectors of the MPCA are calculated and work stages are identified through observing changes in eigenvectors values, as shown in Figure 29.

In addition, the construction stages are identified as generators of unusual structural behaviour since they cause change in the correlations between deformation sensors. Work during the construction stage modify the deformed shape and thus the regression lines between compared sensors shift or change slope. An example is shown in Figure 30. The measurements registered after the work stages are the ones inside the confidence interval $( \pm 3 \sigma)$ while the measurements registered during the construction work are outside the interval. The RRA is used for its capability to work in situations of missing data and outliers. 


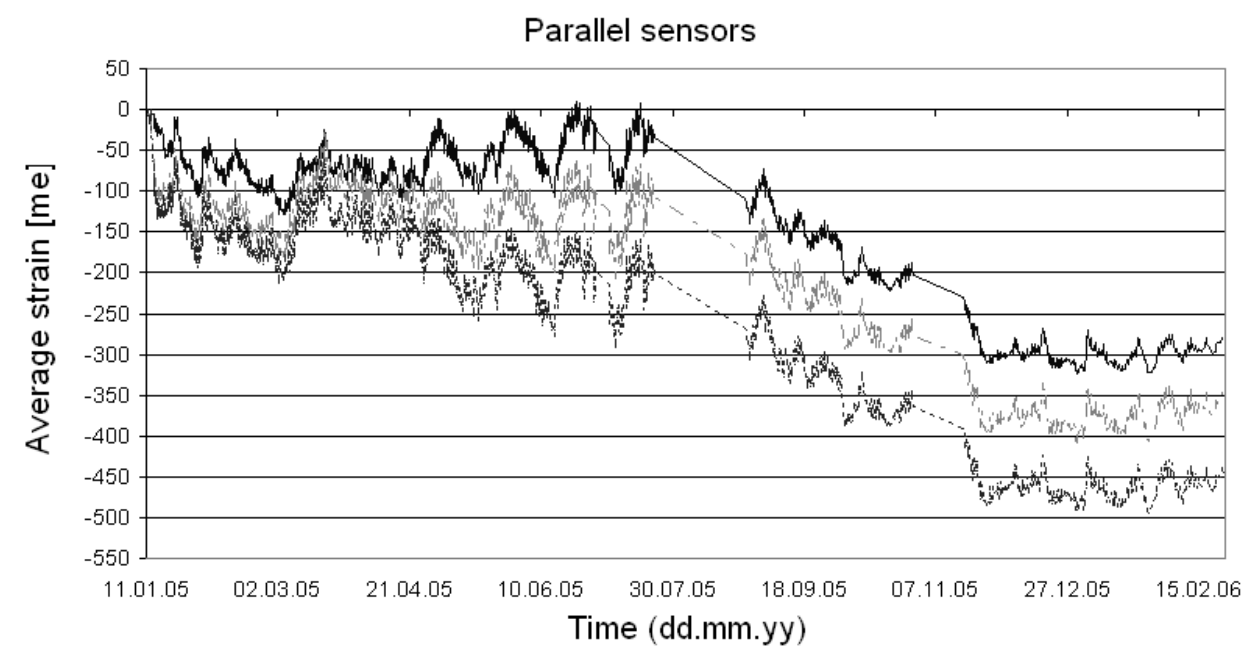

Figure 28: Average strain measured by three parallel sensors. The picture shows a few periods in which the measurements are completely missed.

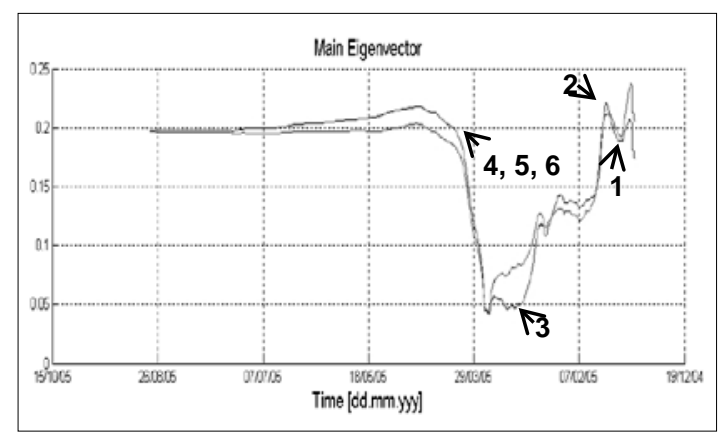

Figure 29: Detection of construction stages as events that create unusual structural behavior (inverted time scale) through the change in eigenvector values.

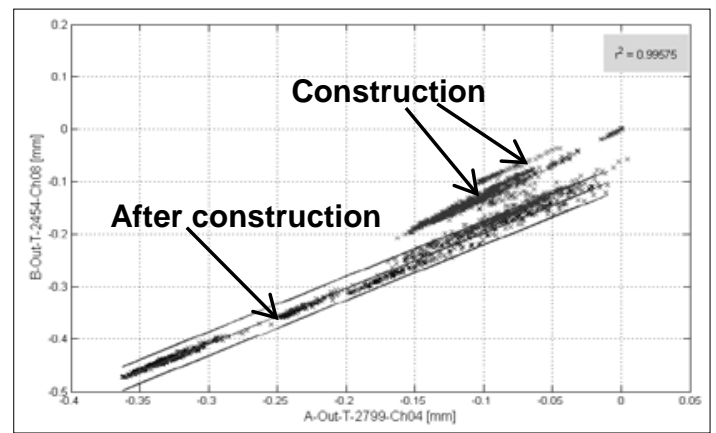

Figure 30: Detection of construction stages as events that create unusual structural behavior through the change in correlation parameters between two deformation sensors.

Another application in which a statistical evaluation of structural behaviour based on

MPCA is performed is the Colle Isarco viaduct on the Italian Brenner-Highway A22. A

detailed description on this bridge and the monitoring system is provided by Bergmeister

and Santa [51]. The bridge is shown in Figure 31. A broad set of sensors are installed, including both traditional and fibre optic sensors (137 SOFO sensors [52]). In this structure, 
the correlation clustering with overlapping algorithm is applied, with the aim to cluster sensors with comparable behaviour and similar sensitivities to damage.

Figure 31 shows an example of two clusters; each cluster contains sensors that are spatially close as well as a few sensors that are spaced well apart. Figure 32 and 33 show the results of the MPCA applied to two clusters after compacting measurement histories due to missing data. In the first plot there are no relevant variations of the eigenvector since there is no damage to the structure. There was no extraordinary maintenance work performed. In the second plot, the time instant when a sensor stopped working properly is detected; the figure shows that only the sensor with the problem has a drift while the others do not indicate changes in the measurements.
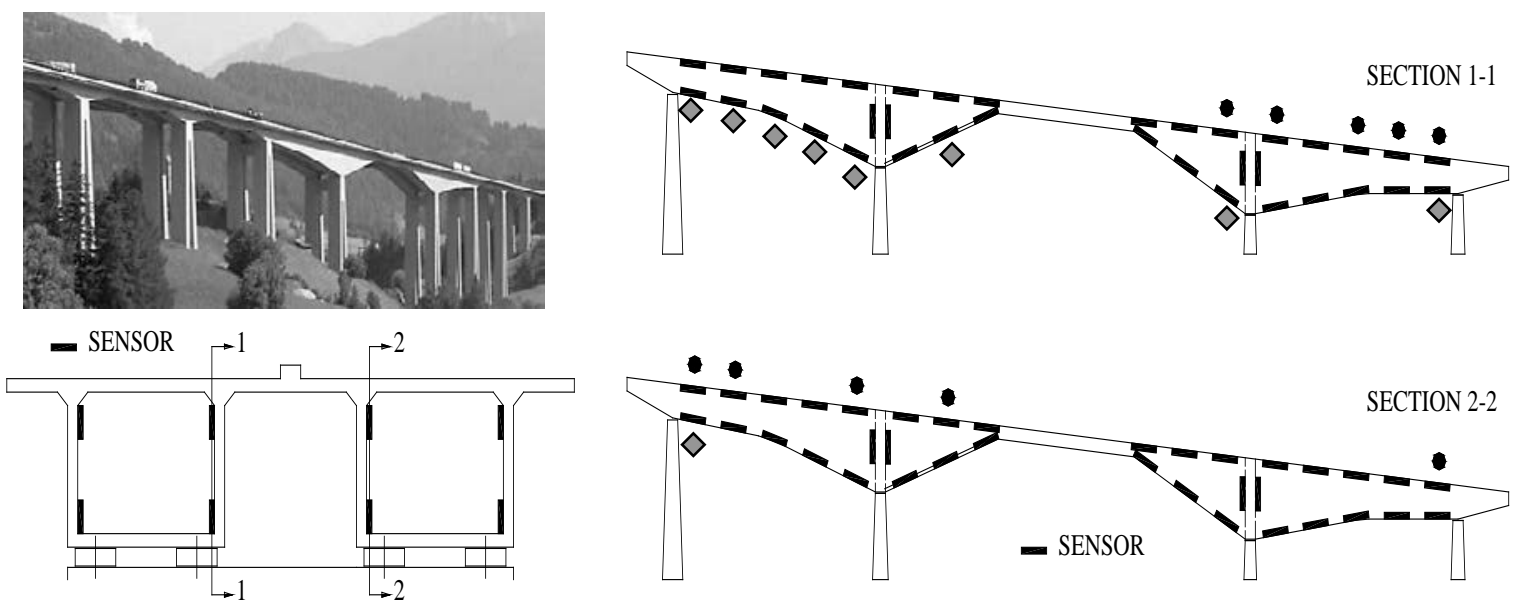

Figure 31: General view, transverse section of the bridge and spatial positioning of sensors along the bridge clusters marked with circles and squares. 


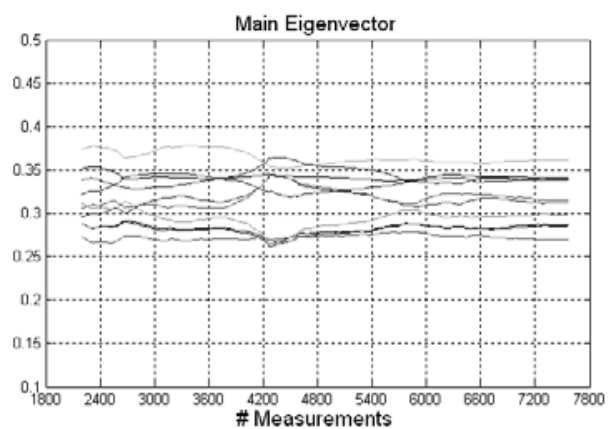

Figure 32: Plot of the main eigenvector of a cluster.

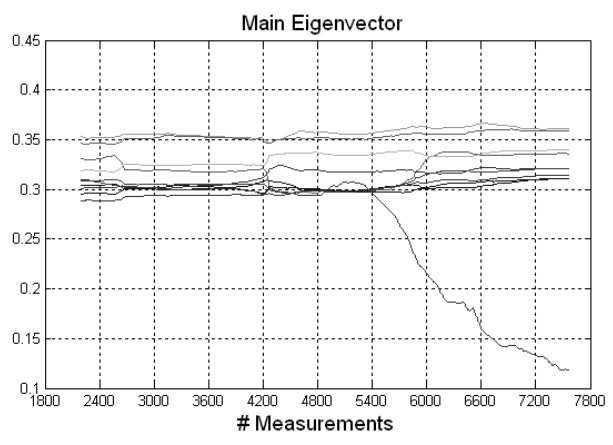

Figure 33: Plot of the main eigenvector of a cluster containing a damaged sensor.

In both examples, problems of missing data, outliers and too many sensors are present. The extended MPCA is successfully applied in both monitoring projects. In the Ricciolo viaduct we are able to identify work stages while for the Colle Isarco viaduct only a damaged sensor is detected and this is the only relevant event. No false positives are detected in either of the case studies.

\section{Conclusions}

Conclusions from this study are the following:

- $\quad$ Moving Principal Component Analysis (MPCA) and Robust Regression Analysis (RRA) are useful tools for identifying and localizing anomalous behaviour in civil engineering structures. These approaches can be applied over long periods of time to a range of structural systems in order to discover anomalous states even when there are large quantities of data. 
- For quasi-static monitoring of civil structures with noise in measurements, MPCA and WPT perform better than wavelet methods, auto regressive analysis, instancebased methods and correlation anomaly scores.

- Robust regression has shown lower performance than MPCA and wavelet analysis but has the advantage of being insensitive to outliers and missing data. Both MPCA and RRA are capable of detecting and locating damage while requiring low computational resources. Another important characteristic is their adaptability. Once new behavior is identified, adaptation allows detection of further anomalies.

- MPCA combined with Interquartile Range Analysis is an effective method when there are outliers in the measurements.

- MPCA can also accommodate situations when measurements are missing completely and when a single measurement is missing.

- A clustering algorithm extends the use of MPCA to those applications where a significant number of sensors are present in the structure. The clustering algorithm uses the correlation between the sensors in order to cluster and gather the sensors with comparable trends and similar damage sensitivities, thus allowing the MPCA to remain tractable for full scale applications.

Conclusions have been validated on two full-scale structures. The results from this study also raise several new issues. Aspects that need to be further investigated include: the definition of the training set, the size of the moving window and the thresholds to use for damage detection. In this study, these parameters are selected on the basis of preliminary 
tests. A more robust strategy that systematically estimates these parameters is needed. Future research could also focus on the use of model-free data interpretation techniques for the analysis of heterogeneous data sets.

Finally, synergies with model-based methods [53] could be identified. Although modelbased approaches are more expensive, they provide high quality support for management decision making related to capacity assessment, replacement avoidance, strengthening following functional changes (for example, changes in loading, widening for extra lane, additional transit uses, etc.) as well as measurement system design [54]. Combining the two approaches is the subject of current research activities.

\section{Acknowledgements}

This work was funded in part by Swiss Commission for Technology and Innovation under Contract number CTI-8613-1. The authors are grateful to Akhilesh Gupta, Francesca Lanata and Branko Glišić for their contributions.

\section{References}

[1] Glišić, B., and Inaudi, D., (2008). Fibre Optic Methods for Structural Health Monitoring, John Wiley \& Sons.

[2] Sohn, H., Czarneski J.A., and Farrar C.R., (2000). Structural Health Monitoring Using Statistical Process Control, Journal of Structural Engineering, 126(11), 1356-1363. 
[3] Lanata, L., and Del Grosso A., (2004). Damage detection algorithms for continuous static monitoring: review and comparison. Proceeding of 3rd European Conference of Structural Control, Wien, Austria, John Wiley \& Sons.

[4] Moyo P., and Brownjohn, J.M.W., (2002). Detection of anomalous structural behaviour using wavelet analysis. Mechanical Systems and Signal Processing, 16(2-3), 429-445.

[5] Sun, Z., and Chang, C.C., (2002). Structural Damage Assessment Based on Wavelet Packet Transform, Journal of Structure Engineering, ASCE, 128(10), 2002, 1354-1361. [6] Han, J.G., Ren, W.X., and Sun, Z.S., (2005). Wavelet packet based damage identification of beam structures. Int. J. Solids Struct., 42,. 6610-6627.

[7] Guo, J., Chen, Y., and Sun, B.N., (2005). Experimental study of structural damage identification based on WPT and coupling N, Journal of Zhejiang University. Science A. 6A(7), 663-669.

[8] Omenzetter, P., Brownjohn, J.M.W., and Moyo, P., (2004). Identification of unusual events in multi-channel bridge monitoring data, Mechanical Systems and Signal Processing, 18, 409-430.

[9] Farrar, C.R., Sohn, H., and Robertson, A. N. (2004). Applications of Nonlinear System Identification to Structural Health Monitoring, in Proceeding 2nd European Workshop Structural Health Monitoring, 59-67.

[10] Omenzetter, P., and Brownjohn, J.M.W., (2006). Application of Time Series Analysis for Bridge Health Monitoring, Smart Mater. Struct. 15, 129-138.

[11] Lu, Y., and Gao, F. (2006). A novel time-domain auto-regressive model for structural damage diagnosis. Journal of Sound and Vibration, 291(1-2), 349-368. 
[12] Nair, K.K., Kiremidjan, A.S., and Law, K.H., (2006). Time series based damage detection and localization algorithm with application to the ASCE benchmark structure Journal of Sound and Vibration, 291(1), 349-368.

[13] Wang, Z., and Ong, K.C.G., (2008). Autoregressive coefficients based Hotelling's T2 control chart for structural health monitoring, Computers and Structures 86(19-20), 19181935.

[14] Deraemaeker, A., Reynders, E., De Roeck G., and Kullaa, J., (2008). Vibration-based structural health monitoring using output-only measurements under changing environment, Mechanical Systems and Signal Processing, 22, 34-56.

[15] Posenato, D., Lanata, F., Inaudi, D., and Smith, I.F.C., (2008). Model-free data interpretation for continuous monitoring of complex structures, Advanced Engineering Informatics, 22 (1), 135-144.

[16] Cavalini Jr., A.A., Franco, V.R., Gonsalez, C.G, Lopes Jr., V., and De Melo, G.P. (2008), Noise Influence On Damage Detection Through Modal State Observers Methodology. Tema Tend. Mat. Apl. Comput., 9(2), 195-204.

[17] Galvanetto, U., and Violaris, G., (2007). Numerical investigation of a new damage detection method based on proper orthogonal decomposition, Mechanical Systems and Signal Processing 21, 1346-1361

[18] Bakhtiari-Nejad, F., Rahai, A., and Esfandiari, A. (2005), A structural damage detection method using static noisy data, Engineering Structures, 27(12), 1784-1793. [19] Wei, W., and Tang, Y, (2003). A Generic Neural Network Approach for Filling Missing Data in Data Mining, IEEE International Conference On Systems Man And Cybernetics 1, 862-867. 
[20] Emam, K.E., and Birk, A., (2000). Validating the ISO/IEC 15504 measure of software requirements analysis process capability, IEEE Trans. Software Engineering. 26(6), 541566

[21] Schafer, J.L., and Graham, J.W., (2002). Missing data: Our view of the state of the art, Psychological Methods, 7(2), 147-177.

[22] Song, Q., and Shepperd, M., (2007.), A New Imputation Method for Small Software Project Data Sets, Journal of Systems and Software, 80(1), 51-62.

[23] Hruschka, E. R., Hruschka JR., E.R., and Ebecken, N.F.F., (2003). Evaluating a Nearest-Neighbor Method to Substitute Continuous Missing Values. Australian Joint Conference on Artificial Intelligence, Perth. LNAI 2903. 723-734.

[24] Batista, G.E.A.P.A., and Monard, M.C., (2003) .An analysis of four missing data treatment methods for supervised learning. Applied Artificial Intelligence 17, 519-533. [25] Jönsson, P., and Wohlin, C., (2006). Benchmarking k-nearest neighbour imputation with homogeneous Likert data Source, Empirical Software Engineering, 11(3), 463-489. [26] Feng, H.A.B., Chen, G.C., Yin, C.D., Yang, B.B., and Chen, Y.E., (2005). A SVM regression based approach to filling in missing values. Knowledge-Based Intelligent Information and Engineering Systems (KES05). Lecture Notes in Computer Science 3683, 581-587.

[27] Arteaga, F., and Ferrer, A., (2005). Framework for regression-based missing data imputation methods in on-line MSPC, Journal of Chemometrics, 19(8), 439-447.

[28] Sharma, S.C., and Allipuram, R., (1993). Duration and Frequency of Seasonal Traffic Counts. Journal of Transportation Engineering, 119(3), 344-359. 
[29] Zhong, M., and Sharma, S., (2006). Matching Hourly, Daily, and Monthly Traffic Patterns to Estimate Missing Volume Data, Transportation Research Record: Journal of the Transportation Research Board, 1957, 32-42.

[30] Barret, V., and Lewis, T., (1994).Outliers in Statistical Data, 3rd Edition, John Wiley

[31] Burke, S., (2005). Missing Values, Outliers, Robust Statistics \& Non-parametric

Methods, LCGC Europe Online Supplement, statistics and data analysis, 14(2),19-24.

[32] Penny, K.I., and Jolliffe. I.T.M., (2001). A comparison of multivariate outlier detection methods for clinical laboratory safety data, The Statistician, 50(3), 295-308.

[33] Ben-Gal, I. E., (2005). Outlier Detection. The Data Mining and Knowledge Discovery Handbook, 131-146.

[34] Yenduri, S., and Iyengar, S.S., (2007). Performance evaluation of imputation methods for incomplete datasets. International Journal of Software Engineering, 17(1), 127-152. [35] Glišić, B., Posenato D., and Inaudi, D., (2007). Integrity monitoring of an old steel bridge using fiber optic distributed sensors based on Brillouin scattering, Proceedings of the SPIE, 6531, 65310P.

[36] FIGG Bridge Engineers. (2008). Bridging the Mississippi; the new I-35W bridge, Judith Dupre.

[37] Lanata, F., and Posenato, D., (2007). A multi-algorithm procedure for damage location and quantification in the field of continuous static monitoring of structures, Key Engineering Materials, 347, 89-94.

[38] Omenzetter, P., and Brownjohn, J.M.W., (2004). Application of time series and Kalman filtering for structural health monitoring of a bridge, Proceeding of 2nd European Workshop on Structural Health Monitoring, Munich, Germany, 836-843. 
[39] Brownjohn, J.M.W., Moyo, P., Omenzetter, P., and Chakraboorty, S., (2004).

Interpreting data from bridge performance and health monitoring systems. Proceedings of the 2nd International Conference on Bridge Maintenance, Safety and Management, Kyoto, Japan, 561-572.

[40] Farrar. C.R., Worden, K., Todd, M.D., Park, G., Nichols, J., Adams, D.E., Bement, M.T., Fairnholt, K. (2007). Nonlinear System Identification for Damage Detection, Los Alamos National Laboratory Report, LA-14353.

[41] Hou, Z., Noori, M., and St. Amand, R., (2000). Wavelet-based approach for structural damage detection. J. Eng. Mech. Div., Am. Soc. Civ. Eng., 12(7), 677-683.

[42] Daubechies, I., (1992). Ten Lectures on Wavelets, Philadelphia: Society for Industrial and Applied Mathematics, 357.

[43] Maeck, J., De Roeck, G. (2003). Damage assessment using vibration analysis on the Z24-Bridge. Mechanical Systems and Signal Processing, 17(1), 133-142.

[44] Nethal, J., (2005). A Review of Robust Regression and Diagnostic Procedures in Linear Regression, Acta Mathematicae Applicatae Sinica, 21(2), 209-224.

[45] Andersen, R., (2008). Modern Methods for Robust Regression. Sage University Inc, 152.

[46] Berry, M., and Linoff, G., (2000). Mastering Data Mining, John Wiley \& Sons.

[47] Witten, I. and Eibe, F., (2005). Data Mining: Practical Machine Learning Tools and Techniques, 2nd Edition, Morgan Kaufmann, ISBN 0120884070.

[48] Idé, T., Papadimitriou, S., and Vlachos M., (2007). Computing Correlation Anomaly Scores using Stochastic Nearest Neighbors, Omaha, USA, ICDM 2007, 523-528. 
[49] Lanata, F. (2005). Damage detection algorithms for continuous static monitoring of structures, PhD Thesis, University of Genoa, Italy.

[50] Glisic, B., Posenato D., Inaudi, D., and Figini, A., (2008). Structural health monitoring method for curved concrete bridge box girders, Proceedings of the SPIE, 6932, 693204.

[51] Bergmeister, K., Santa, U. (2000). Global monitoring concept for bridges. Proceedings SPIE, 3995, 14-25.

[52] Inaudi, D., and Glisic, B., (2002). Long-gage sensor topologies for structural monitoring, 1st fib Congress on Concrete Structures in the 21st Century, Osaka, Japan. 2, Session 15, 15-16, on conference CD.

[53] Smith, I.F.C. and Saitta, S. (2008) "Improving Knowledge of Structural System Behavior through Multiple Models" Journal of Structural Engineering, Vol 134, No 4, pp 553-561.

[54] Kripakaran, P. and Smith, I.F.C. (2009). Configuring and enhancing measurement systems for damage identification, Advanced Engineering Informatics, Vol 24, No 4. 
Table 1 Results of a comparative study between the algorithms using data derived from a numerically simulated beam in healthy and damaged states. $\mathrm{D}=$ detected, $\mathrm{N}=$ not detected

\begin{tabular}{|c|c|c|c|c|c|c|c|c|c|c|c|c|c|}
\hline \multirow{2}{*}{ Algorithms } & \multicolumn{13}{|c|}{ Damage scenario } \\
\hline & A1 & A2 & A3 & A4 & B1 & $\mathrm{B} 2$ & B3 & B4 & B5 & B6 & B7 & B9 & B10 \\
\hline ARMA & $\mathrm{D}$ & $\mathrm{N}$ & $\mathrm{N}$ & $\mathrm{N}$ & $\mathrm{N}$ & $\mathrm{N}$ & $\mathrm{N}$ & $\mathrm{N}$ & $\mathrm{N}$ & $\mathrm{N}$ & $\mathrm{N}$ & $\mathrm{N}$ & $\mathrm{N}$ \\
\hline Box-Jenkins & $\mathrm{D}$ & $\mathrm{N}$ & $\mathrm{N}$ & $\mathrm{N}$ & $\mathrm{N}$ & $\mathrm{N}$ & $\mathrm{N}$ & $\mathrm{N}$ & $\mathrm{N}$ & $\mathrm{N}$ & $\mathrm{N}$ & $\mathrm{N}$ & $\mathrm{N}$ \\
\hline Seasonal ARIMA & $\mathrm{D}$ & $\mathrm{N}$ & $\mathrm{N}$ & $\mathrm{N}$ & $\mathrm{N}$ & $\mathrm{N}$ & $\mathrm{N}$ & $\mathrm{N}$ & $\mathrm{N}$ & $\mathrm{N}$ & $\mathrm{N}$ & $\mathrm{N}$ & $\mathrm{N}$ \\
\hline DWT & $\mathrm{D}$ & $\mathrm{D}$ & $\mathrm{D}$ & $\mathrm{D}$ & $\mathrm{D}$ & $\mathrm{D}$ & $\mathrm{D}$ & $\mathrm{N}$ & $\mathrm{N}$ & $\mathrm{N}$ & $\mathrm{N}$ & $\mathrm{N}$ & $\mathrm{N}$ \\
\hline WPT & $\mathrm{D}$ & $\mathrm{D}$ & $\mathrm{D}$ & $\mathrm{D}$ & $\mathrm{D}$ & $\mathrm{D}$ & $\mathrm{D}$ & $\mathrm{D}$ & $\mathrm{D}$ & $\mathrm{D}$ & $\mathrm{D}$ & $\mathrm{D}$ & $\mathrm{D}$ \\
\hline RRA & $\mathrm{D}$ & $\mathrm{D}$ & $\mathrm{D}$ & $\mathrm{D}$ & $\mathrm{D}$ & $\mathrm{D}$ & $\mathrm{N}$ & $\mathrm{N}$ & $\mathrm{N}$ & $\mathrm{N}$ & $\mathrm{N}$ & $\mathrm{N}$ & $\mathrm{N}$ \\
\hline IBM & $\mathrm{D}$ & $\mathrm{D}$ & $\mathrm{D}$ & $\mathrm{D}$ & $\mathrm{D}$ & $\mathrm{D}$ & $\mathrm{D}$ & $\mathrm{D}$ & $\mathrm{D}$ & $\mathrm{D}$ & $\mathrm{N}$ & $\mathrm{N}$ & $\mathrm{N}$ \\
\hline \multicolumn{14}{|l|}{ Correlation } \\
\hline Anomaly Scores & $\mathrm{D}$ & $\mathrm{D}$ & $\mathrm{D}$ & $\mathrm{D}$ & $\mathrm{D}$ & $\mathrm{D}$ & $\mathrm{D}$ & $\mathrm{D}$ & $\mathrm{N}$ & $\mathrm{N}$ & $\mathrm{N}$ & $\mathrm{N}$ & $\mathrm{N}$ \\
\hline \multicolumn{14}{|l|}{ Analysis } \\
\hline MPCA & $\mathrm{D}$ & $\mathrm{D}$ & $\mathrm{D}$ & $\mathrm{D}$ & $\mathrm{D}$ & $\mathrm{D}$ & $\mathrm{D}$ & $\mathrm{D}$ & $\mathrm{D}$ & $\mathrm{D}$ & $\mathrm{D}$ & $\mathrm{D}$ & $\mathrm{D}$ \\
\hline
\end{tabular}

This work is licensed under a Creative Commons Attribution-NonCommercialNoDerivatives 4.0 International License

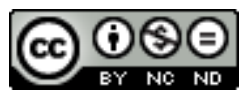

